\title{
Mener Baltäbitider.
}

$$
\left[n_{r} .4 i 0\right]
$$

ditn $\mathbb{C} \mathfrak{i} \mathfrak{l}$

Ẽin Sommernadytstraum

volt

\section{şeituridj şeitte.}

\section{Motto:}

2uకి Dem iđjimmernden tweiḡen 3elte hervor

Iritt ber (d)lad)tgeriijtete füritlidje Mobr:

So tritt aus jdimmernder $\mathfrak{B o l f e n}_{\text {Ihor }}$

Der Monto, Der vecimiterte, Dunfle, herbor.

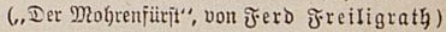

Tripriy und Antent.

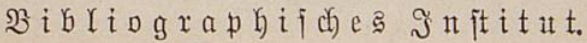




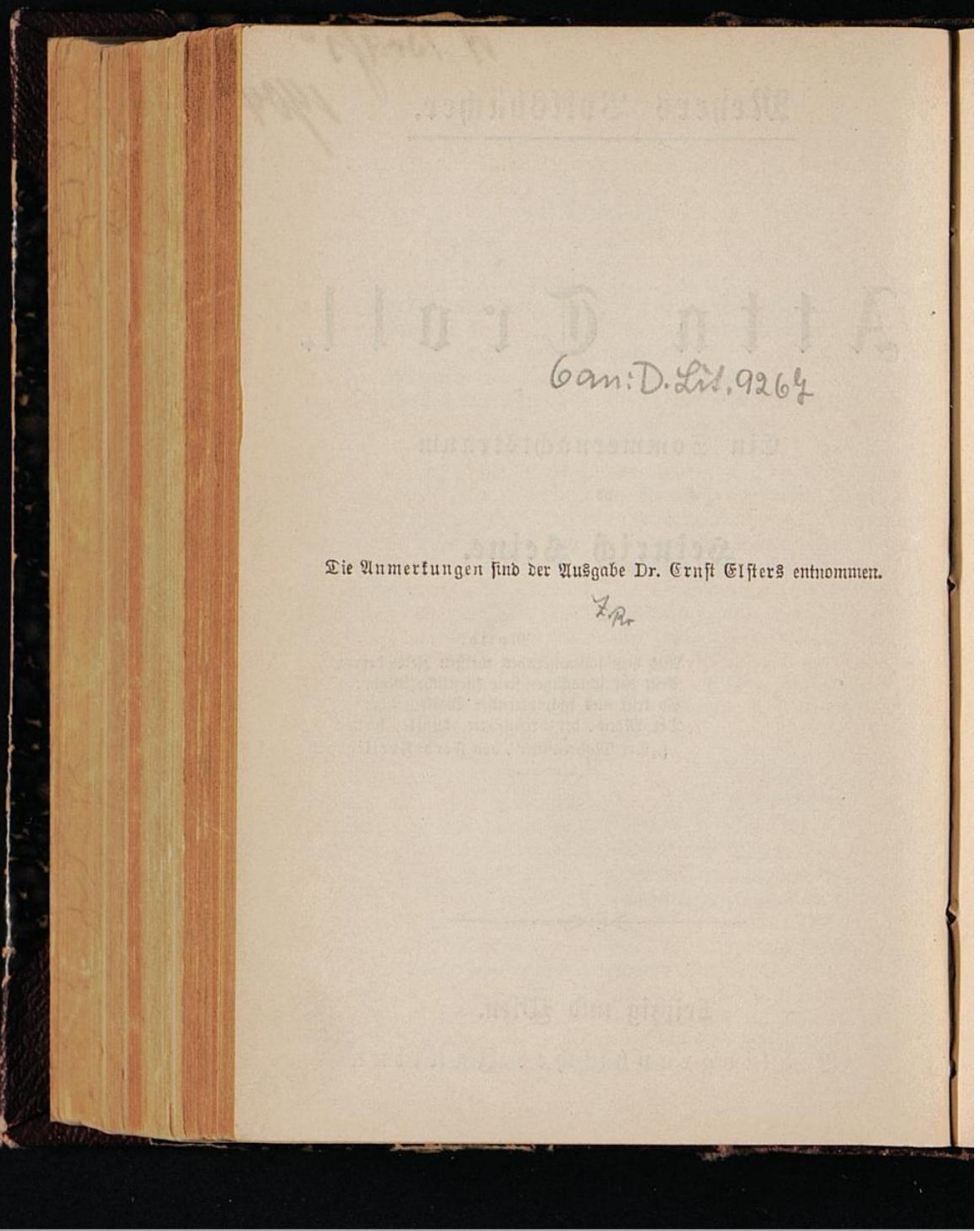

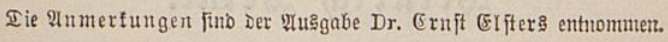

$$
\text { X.pr }
$$




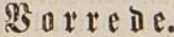

Der "ŶAtta Troll" entitand im Spätherbite 1841 und mard fragmentarijc) abgebuclt in ber "Eleganten Welt", alङ meit Freund Seinrich saube wieder Die Rebaftion Derjelben über: nommen hatte. Intalt und Bujchnitt bes (5) edicjtes muiten Den zabmen Bebürfnifjen jenex Beitichrift entiprecjen; idj iarieb vorläufig mux die Rapitel, bie gebrud werden fonnten, und auch Dieje exlitten manche Bariante. \$ch hegte bie $2(b=$

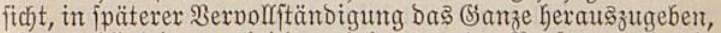
aber es blieb immer bei dem lobensmerten Borjabe, und wie

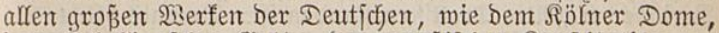

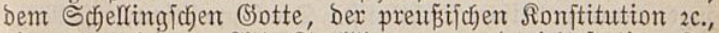

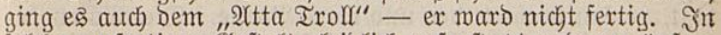

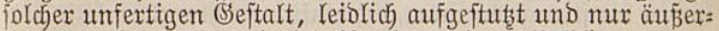
(ich) gerünbet, übergebe ich ifn heute Dem \$ublico, eintem Drange gehorchend, ber mahrlich nidjt won innen fommt.

Der 2 tta Iroll entitand, wie gejagt, im Spätherbite 1841, 3u einer Beit, als bie grop̃e Emente, two bie verjotedenfarbig. iten Feinde fich gegen mich zujammengerottet, noch nicht ganj ausgelärmt hatte. (5) war eine jebr grop̉e (smente, und ici) Gätte nie geglaubt, dafi Deutichland io viele faule Silpfel her: vorbringt, wie mir Damals an Den fopf flogen! Itnjer $\$$ ater:

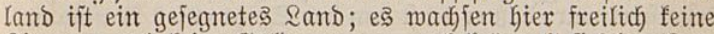
Bitronen und feine (Solororangen, auch früppelt fich) ber Ior=

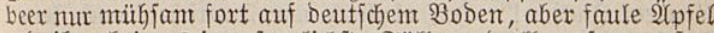
gedeifen bei unt in erfreultichfter Fülle, uno alfe unfere gropen Didjter musten bavon ein Ried zu fingen. Bei jener Emeute, wo ich Rrone und Ropf verlieren follte, perlor $i$ ch feins vour beiden, und bie abjurben 2(njchuldigungen, momit man ben

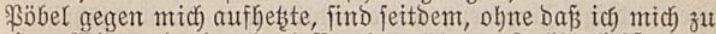
einer \$idoerrebe herabzulaffen braudjte, auf̧ fläglichfte vex= icholfen. Die Beit übernahm meine Rechtfertigung, und audf Die refpeftiven bentichen Regienungen, ich mū́ es banfbar an= erfentren, Gaben fitch in biejer Beziebung verdient um mich ge= 


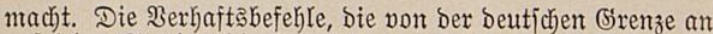
auf jeder Station Die Seimfehr beకె Didyters mit Sebnjucht ermarten, werben gehörig renvviert jebes $\Im a h x$, um bie heilige Weifnachtgzeit, wenn an Den Shriftbäumen bie gemütlichen

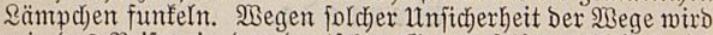
mir bas Reijen in Den Deutjcjen (sauen fojier verleidet, id feiere Deşhalb meine \$eihnachten in Der Frembe, und werbe auch in ber Frembe, im Exil, meine Tage bejaliešen. Die

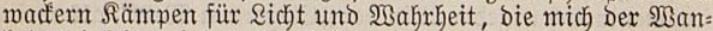

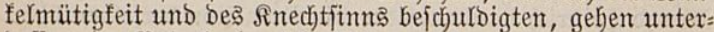
Deffen im Baterlande fehr ficher umber, als wohlbeftallte

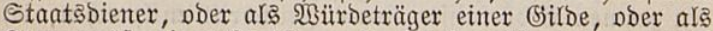
Stammgäfte eines Rlubs, wo fie fich) Des 2(bends patriotifa)

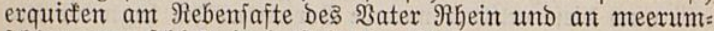

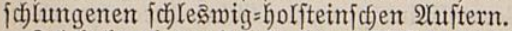

Jch habe oben mit bejonderer $2(b$ ficht angebeutet, in meldyer \$eriobe Der "2ltta Troll" entitanden ift. Damals Glühte Die jogenannte politifje Dichtfunjt. Die Dppofition, wie Ruge fagt, verfaufte ifr Reder und mard \$oefie. Die Mujen be=

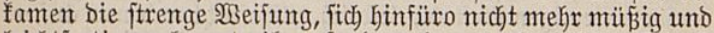
leichtfertig umberzutreiben, fonbern in vaterlänoifçen Dienft zu treten, etwa alక Marfetenderinnen ber Freiheit ober als

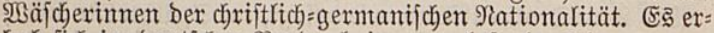
hub fich im Deutichen Bardenthain ganz bejonders jener vage, unfuchtbare \$atbos, jener nublofe Enthuftasmusbunft, Der (ich) mit Tobesperachtung in einen Dzean von 2UMgemeinheiten ftürzte und mich immer an ben amerifanij chen Matrojen er= innerte, welcher für ben (Śeneral Jacfjon fo überichmenglid begeiftert mar, baj ex einft von ber Spize eines Maftbaum ins Meer binabiprang, inbem er ausrief: "Ĩc) fterbe für ben General Jadfjon!" Ja, obgleich wir Deutichen noch feine Flotte bejajen, io batten wir bod) fdyon viele begeifterte Ma: trojen, bie für ben Seneral Jadfion ftarben, in Jerjen uno in \$roja. Das Talent war Damals eine jebr mif̧liche Begabung, Denn es bracbte in Den \$erbacht Der Eharafterlofitgfeit. Die idjeeljüchtige Jmpotenz Gatte enolich nach taujendjährigem Nad)grübeln ihre grofe $\mathfrak{B}$ affe gefunden gegen Die übermüben Des S'enius; fie fant nämlict) die 2lntitheje von Ialent und (Eharafter. Es war faft perjönlich fdymeichelfaft für bie grofie Menge, wemn fie behaupten hörte: bie braven Seute feien frei: 


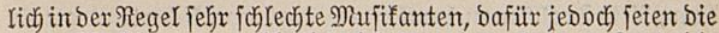

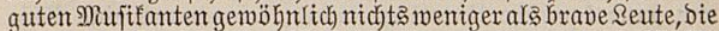
Brankeit aber fei in ber Selt bie Sauptjacje, nicht bie Mujif. Der leere Ropf pochte jebt mit Fug auf jein volles Şerz, und bie (Seftnmung mar Irumpf. Sib) erintere mid) eines bama=

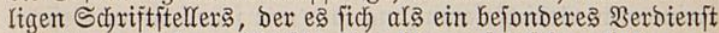

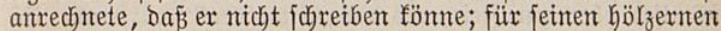
Stil befam ex einen filbernen (s)

Bei Den emigen Sb̈̈ttern! Damals galt es die unveräuñer=

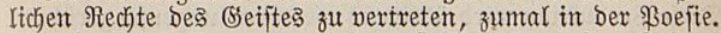

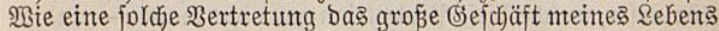
war, io babe id fie am allermentigiten im vorfiegenden (b)e= bicht auñer 2(ugen gelaffen, und jowohl Tonart als Stoff bes=

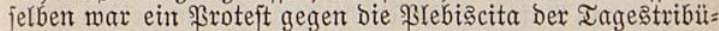
nen. Ind in ber That, fajon bie erften Fragmente, bie vom

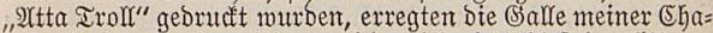
rafterbelden, meiner $\Re$ ömer, bie mich nicht blof ber littera= rifchen, jonbern auch Der gejellichaftlichen Reaftion, ia jogar

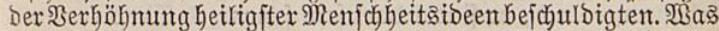
Den äfthetifchen \$Bert meines \$oem betrifft, fo gab ich ifn gern preis, wie ich es auch Geute noch thue; ich ichrieb bas = jelbe zu meiner eignen \&ujt und Freube, in ber griflentaften Traummeije jener romantijcjen Schule, wo ich meine ange= nefmiten Jugenbjahre verlebt, und zulebst ben Schulmeijter geprïgelt habe. Jn biejer Beziehung ift mein (Sebicht viel= leicht vermerflich. 2(ber bu lügit, Brutus, Du lügft, (Eaffitus, uno aud) bu lügit, 2(jinius, wenn ihr behauptet, mein Spott träfe jene Joen, Die eine foftbare Errungenichaft ber Menj(d)= heit find und für bie ich jelber jo viel gejtritten und gelitten habe. Nein, eben meil bem Didjter jente Joen in Gerrlicjiter

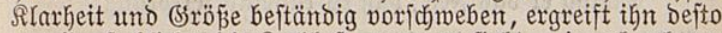
unmiberftehlicher bie \&achluft, menn er fieht, wie roh, plump

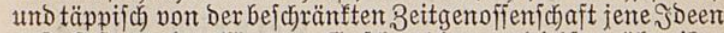

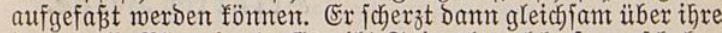
temporelfe Bärenhaut. (Ẻ gibt Spiegel, welche jo verjd)oben

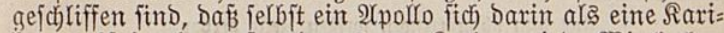

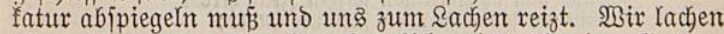

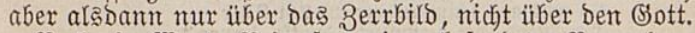

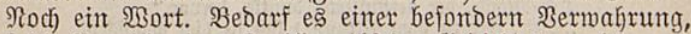

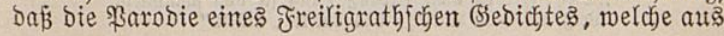




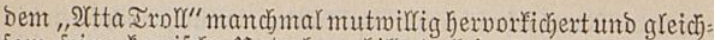
fam jeine fomifiche Interlage bildet, feineswegg eine Mitis=

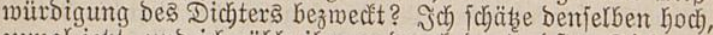
zumal jest, und ich zähle ibn zu ben bebeutenditen Didjtern, Die feit Der Эulius?revolution in Deutichland aufgetreten fins. Seine erfte Sebichtejammlung fam mix fehr ppät ơ Seficht,

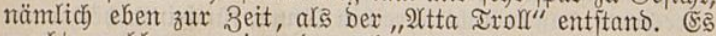
mod)te mohl an meiner bamaligen Stimmung liegen, bafis namentlich Der "MTohrenfürit" io beluftigend auf mid' wirfte. Dieje \$roduftion wird übrigens als bie gelungenfte gerübmt.

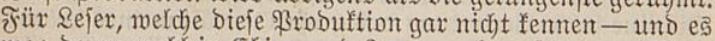
mag beren wohl in Shina und Japan geben, fogar am Niger uno an Sentegal - für bieje bemerfe ich, baf̈ Der Mohren= fönig, Der zu 22nfang Des S'ebichtes aus feinem meifpen Belte, wie eine Monofinfternis ${ }^{1}$, hervortritt, auch eine fdb waxze G'e= liebte befist, über Deren Dunfles 2Tntlibs Die weipen Straubs= febern nidfen ${ }^{2}$. 2Uber friegsmutig verläß̈t er fie, er zieht in bie Rigerjaladjt, wo da raffelt Die Irommel, mit Scjäbeln behangen - ach), ex finbet bort jein jabarzes saterloo und

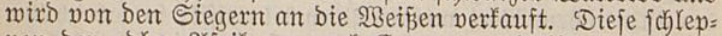
pen ben eblen 2lfrifaner nach) Eurppa, und bier finden wir ifn wieber im Dienfte einer Kerumziehenben Reutergejellichaft, bie ifm bei ihren Sunftuorftellungen Die türfifche Trommel anvertraut hat. Da ftebt er mun, finfter uno ernithaft, ant

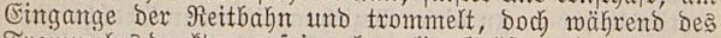
Trontmelns Denfter an feine ehemalige S'röje, er Dentt baran, Daj er einft ein abjoluter Monard war am fernen, fernen Niger, uno baf́s ex gejagt Den Römen, Den Iiger -

"Sein 2Utge mard naf;; mit bumpfem Rlang "Scjlug er das Jell, Dẩ es raffelnd zeriprang."

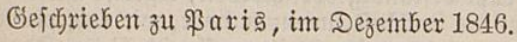

\section{Şeitrid Şeitue.}

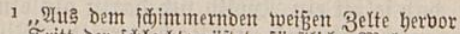

Iritt Der id)laditgeriiftete füritlidje Mohr;

So tritt aus fojimmernder 230 lfen Thor

Der M2ono, Der verfinterte, Dunfle, bervor."

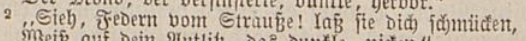

M̉eî auf Dein 2uttlib, Das Dunfle, nicten." 


\section{æaput $\mathrm{I}$.}

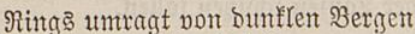
Die fich trotsig ïbergipfeln, Itnd von wilden Bafferitürzen singelulfet, wie ein Iraumbilo,

Riegt im Ihal bas elegante Eautereta. Die meifien Şäuschen Mit Balfonen; idjöne Damen Stehn barauf und lachen heržlich.

Serzlich Iachento fochaun fie niebec 2(uf Den wimmelno bunten Marftplais, WD Da tanzen Bär und $\mathfrak{B a ̈ r i n}$ Bei bes Dubeljactés Rlängen.

Ytta Ixoll und feine S'attin, Die geheifipen f(d)warze Mumma, Sino Die Tänzer, und ę jubeln

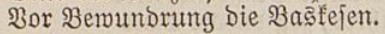

Steif und exnithaft, mit Sirandejan, Tanzt Der edle eltta Iroll, Doch Der zott'gen (Ehehärffte Fehlt bie \$ürde, fehlt Der 2 (nnftand.

Ja, eకె will midf f(fier bebünften, Daf fie manchmal cancaniere, und gemtütlos frechen Steip̄purfs

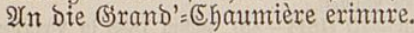

2(uc) Der wad́re Bärenführer, Der fie an Der Rette leitet, Scheint Die Immmoralität Shrę Tanzes zu bemerfen. 
Und er langt ifr manchmal über Ein'ge Şiebe mit Der Peitjche, Und Die fdjmarze Mumma heult Dann, Dap̉ die ßBerge miberballen.

Diejer Bärenführer trägt Sechs Mabonnen auf Dem Spib̨hut, Die jein Saupt vor Jeindesługeln Doer \&äujen joüüzen jollen.

\section{über jeine Schulter hängt} Fine bunte 2rltardecfe, Die als Mantel fich gebärobet; Drunter Iaujcht Bijtol und Meffer.

Mुar ein Mänch in jeiner Jัugent, Später ward ex Räuberhauptmann; Beibes zu verein'gen, nahm ex Endrich) Dienjte bei Don Siarlos. ${ }^{1}$

थrs Don Raulos flieben muĩte Mit Der ganzen Tafelmunde, Und bie meiften Palabine

Nach Gonettem Şandwerf griffen -

(5err Schnapphansfit murbe $2(u$ tor) Da mard unjer ślaubensiritter Bärenfüthrer, zog ourchs \&and Mit bem 2 tta Irolf und Mumma.

Und er läpt bie beiben tanzen 刃or Dem ミolfe, auf Den Märften; -

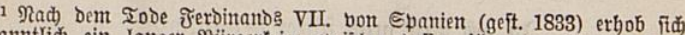
befanntlid) ein Ianger Bürgerfrieg jlvijden vejien jüngerem Bruber Don fiarlog und Der Siönigin = शisitwe Maria (Sbriftina, tveld)e Die Regentjd)aft für ibre Todter Jijabella fïbrte. Don Siarlog bielt fid) zur flerifalen, Maria (E)riftina jur liberalent \$artei.

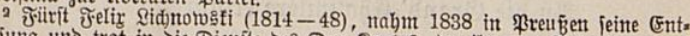
Iajiung und trat in Die Diente Des Don' farlos, Der ibn zum Drigabegenteral und (Seneralabjutanten ernannte. Nad) Der Riidfebr aus Spantien färieb (id)notwgti , Erimnerungen aus Den Jabren 1837-39" (2 פDe., Jranff. 1841 bis 1842), bie ibn in ein Duell berwidelten, in weld)em er fdiwer vermuntet twarb. Befantlidy twurbe er im Eeptember 1848 beim Jranfiuter 2ufitand 
YUf Dem Marft non Eauterets Tambt gefeffelt 2 tta Troll!

Ytta Iroll, ber einjt gehaujet,

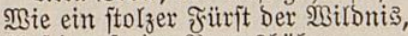
2(uf Den freien \$ergeß̧ด̈hen, Tanzt im Thal vor Mienjadenpöbel!

Itnd jogar für fanöbes Seld Muí er tanzen, er, Der meiland

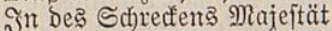
Sidf io melterbaben füblte!

Denft ex jeiner Jugendtage, Der verlornen Waldesherridajt, Dann erbrummen bunfle \&aute Ât Der Seele 2(tta Irolla;

Finfter fachaut ex wie ein fajwarzer Frreiligräthjcher Mohrenfürft, Und wie biejer falecht getrommelt,

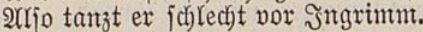

Doch ftatt Mitgefühl erregt er গur Seläd)ter. Selbit J̃uliette Sacht Kerunter vom Balfone Db Den Sprüngen Der Љerzmeiflung. - -

ฐuliette bat im $\mathfrak{B u j e n}$ Rein S'emüt, fie ift Frtanzöfin,

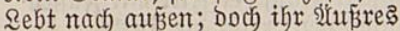
Sitt entzüßfend, ijt bezauberno.

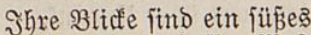
Strablentes, in beffen Majdjen Unjer Şerz, gleich einem fitid)lein, Sid) verfängt und zärtlid) zappelt.

\section{æaxut II.}

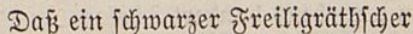
Mohrenfürjt jebnjüchtig loşpauft 
2uf bas Fell ber grop̃en Trommel, $23 i s$ es praffelno laut entzmeifpringt:

Das ift mahrbaft trommelrübrend Itnd aud trommelfellerichütterns U(ber Denft euta) einen 3 ären, Der fich von Der Rette lošreifit!

Die Mufif und Das Selädjter, Sie verítummen, unb mit 2 mafticjuet Stürzt vom Martte fort das গুolf, Und bie Damen, fie erbleichen.

ฐa, von feiner Słlavenfeffel Sat fich plötilich losgeriffen 2ltta Ivoll. Mit milden Sprüngen Durch) Die engen Strapen rennend -

(J̃eber macjt ifm höflich \$Iab) Flettert ex binauf Die Jeljen, Schaut bimunter, mie verhöbnend, Ind verjd) windet im Siebirge.

UUf Dem leeren Marftplabs bleiben Sianz allein bie fdy warze Miumma Und der ßärenfübrer. Rajend Schmeipt ex feinen 5ut zux (5xDe,

Trampelt brauf, ex tritt mit öüpen Die Mabonnen! reift die Decte Sich vom jcheuffich nacften seib, Fluct und jammert über Utrbant, Über \{chmarzen Bärenumbanf! Denn ex babe Atta Troll Stets wie einen Jreund behandelt Und im Tanzen unterridjtet.

2rres hab' ex ifm zu Danfen, Selbit das qeben! Bot man bod Эhim vergebent hundert Ihaler Fux Die Scaut Des 2 tta Irolr! 
2(uf bie arme fdrwarze Mumm Die, ein Bill Des ftummen Srrames, Flehend, auf Den Sintertaken, ßor Dem 5̧ocjerz̈ürnten ftehn blieb,

Fällt Des 5ocherzürnten \$ut

(Enolich Doppelt ichmer, ex icjlägt fie, Rennt fie Rönigin Ehriftine, 2(ud) Jrau 9)?unoz ${ }^{1}$ uno \$utana. - -

Das geidjah an einem fchönen, Wुarmen Sommernad)mittage, Ind bie Radbt, Die jenem Tage Sieblich folgte, wax füperbe.

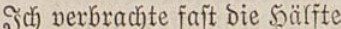
Jener Nacht auf Dem Balfone. Reben mix ftand ฐuliette Und betrachtete die Sterne.

Senfzend iprach fie: ", $2($ (b), bie Sterne Sind am jobonften in \$aris, Wुent fie Dort bes Winterabends In Dem Strafienfot fich ipiegeln."

\section{æaxt III.}

Traum Der Sommernadyt! \$hantaftifh 8 medtlos ift mein Ried. Ja, żwedtos

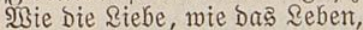
NBie Der Schöpfer famt Der Sçöpfung!

Nur ber eignen suft gehorcheno, Galoppierent ober fliegent, Iummelt fich) im Fabelreiche Mein geliebter \$egajuב.

1 Maria (5)rifina bermählte fid) balo nad) Des \&önigs Tode mit einem

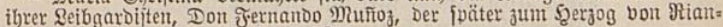
sares erbobent tourde. Ec ftarb 1873. 
Jit fein nübrich tugenohafter Rarrengaul Des Rürgertums, Roch ein Sa)lachtpfers Der \$arteimut, Das pathetific ftampft und wiekert!

(5)

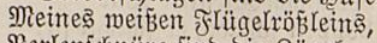
\$erlenjchnüre find bie Bügel, uno ich lafi' fie lujtig jujiefen.

Trage mich, wohin bu willft! l̈ber luftig fteilen Bergpfad, 30 Rasfaden angituoll freijchend

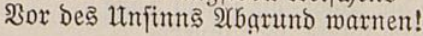

Trage mich ourch ftille rhäler, 20 bie (Eichen exnjthaft ragen Und ben Murzelfnorrn entriejelt Uralt füfer Sagenquelr!

¿á̃ mich trinfen bort unঠ näfjen

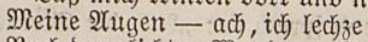
Rach bem licjten Wunbermaffer, ख્ldję jebend madjt und wiffend.

J̃ebe Brinokeit weicht! Mein Blic Dringt bis in bie tieffite Steinfluft, In Die Şöhle 2rtta Iroll -

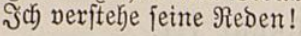

Sonberbar! mie mohlbefannt Dünft mix Dieje Bärenjpraçe! Sab' idj) nicjt in teurer Seimat 厅rtüh vernommen bieje 2aute?

\section{玉apนt IV.}

Ronceval, du edles Thar! Wenn idy Deinen Ramen höre, Bebt und Duftet mir im Sgerzen Die verfdjollne Glaue Blume! 
SSlänzens fteigt empor Die Iraummelt, Die jahrtaujenolich verjunfen, Ind Die gropien S'eifterangen

Schaun mich an, baj ich erjofjecte! !

Itno es flirt und toft! (ङอ fämpfen Sarazen und Franfenritter; פ3ie verzmeifelt, wie verblutend, Rlingen Rolanos Malohornü̈fe!

In Dem Thal von Ronceval, Unfern von Der Rolandsicjarte So geheī̄en, weil ber Seld, Um fich einen $\mathfrak{S e g}$ zu bahnen,

Mit bem guten Scfwert Duranoa 2rfo tobeşgrimmig eintieb İn bie Felsmand, Dap̃ die Spuren Bis auf Geut'gem Iage fichtbar -

Dort in einer büftren Steinichludft, Die ummachjen von Dem \$Bujbuert Bilber Iannen, tief verborgen, Siegt Die ફ̈̈hle 2Ytta Irolls.

Dort, im Schofie ber Familie, Rubt ex aus von Den Strapazen Seiner Fludjt und von ber Mü̈jial Seiner 刃ölferfanau und Beltfahrt.

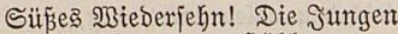
Fanto er in ber teuren Sö̈hle, Wo er fie gezeugt mit Mumma; Söhne vier und T̈̈bjter zmei.

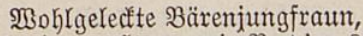
Blono von 5aax, wie \$red'gerstöb)ter; Braun Die \$3uben, nur Der jüngite Mit Dem einz'gen Dhr ift ichmarz.

Diejer jüngfte max bas Serzblatt Seiner Mutter, bie ihm ipielend 2Ybgebiffen einft ein $\mathfrak{D h r}$; und fie frapis es auf vor siebe. 
Sift ein gentaler Эüngling, ร̈̈̈r (Symnajtif jebr begabt, Und ex jablägt Die \$urzelbäume \$ie ber Iurnfunjtmeijter Maß̄mant.

Brüte autochthoner Biloung, Siebt er mur bie Mutteriprache, ¿ernte nimmer Den Jargon

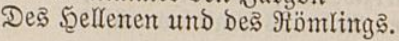

Frifch und frei und fromm und fröblich), Sit verbafit ifm alle Seife, ¿urus bes modernen $\mathfrak{W a j}$ jent, Wie Dem Tumfunjtmeifter Maß̆mann.

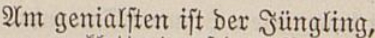
Wenn ex flettert auf Dem Baume, Der entlang Der fteilften Felsmand 2(uS ber tiefen Schlud)t emporiteigt

Und hinaufragt bis zur Soppe, Mুo Des Nachts bie ganze Sippicjaft Sich) verfammelt um ben \$ater, Rojend in der 2(bendfüble.

Sern erzählt alsbann ber 2rite, Was er in ber Belt erlebte, Mie ex Menjchen viel' und Stäbte (sinft gejebn, auch viel erbuldet,

SIleich) Dem edlen saertiaben, Diejem nur barin unäbnlicb, Daj̉ die Saattin mit ifm reijte, Seine jojmarze \$enelope.

2(ucb) erzählt Dann 2(tta Irolf Bon Dem foloffalen Beifall, Den ex einjt Durch jeine Ianzfunft Eingeerntet bei Den Menjcjen.

(sr verfichert, jung und alt Sabe jubelns ifn berundert, 2Benn ex tanzte auf Den Märften Bei ber Sacfifeip' jüpen হönen. 
Und bie Damen ganz Gejonders, Dieje zarten Renterinnen, Sätten rajens applaudiert und ifm huldoreich zugeäugelt.

D, Der Rünttlereitelfeiten! S(f)munzelnd Denft Dex alte Tanzbäx $\mathfrak{A} n$ bie Beit, mo fein Iarent Nor Dem \$ublico fich zeigte.

übermannt von Selbjtbegeiftututg, Mill er burch bie That befunden,

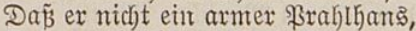
Daf́ ex wirffich grof́ ale Tänzer -

Und vom B̧oben fpringt ex plöt̨)(id), Stellt fitch auf Die Sintertaber, Und wie efmals tanst ex mieder Seinen Reibtanz, Die Savotte.

Stumm, mit aufgepperten Sdyauzen Schauen วu bie Bürenjungen,

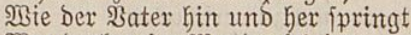
Wunderbar im Mondenjajeine.

\section{æaput $V$.}

In Dex Söhle, bet ben Seinten, Siegt gemütsfranf auf Dem Rüctelt 2tta Iroll, nadjoentlich faugt er 2(n ben Taken, jaugt und brummt: , Mumma, Mumma, fantwarze Perle, Die ich in bem Meer bes Rebent

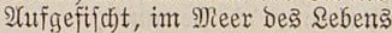
5ab' icf toieder bich verloren!

"2̧erd' ich nie bich twieberjehen, Doer mux jenjetts des Srrabes, Mo von ExDenzotteln frei Sich) verffäret beine Seele? 


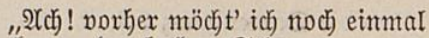
secfen an ber holden Sdmatze ) Neinter Mumma, bie po jübe, \$ie mit \$onigfeim beftrichen!

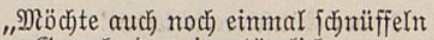
Den (Seruch), Der eigentüm(ic) Meiner teuren fajwarzen Mumma, Und wie Rojenduft fo lieblicb!

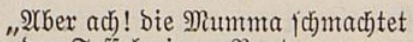
Эn' Den Feffeln jener Brut,

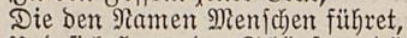
und fich ફerrn ber Schöpfung Dünfelt. "Tod und 5̈̈lle! Dieje Menjçen, Dieje Erzariftofraten, Schaun auf bas gefamte Tierreich శrech) und abelftolz herunter,

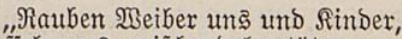

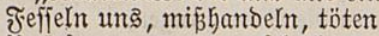
Ins jogar, um zu verjajachern Un nje 5̧aut uno unjern Reichnam!

"Itno fie glauben fich berechtigt, Solche Unthat auszzuüben Şanz bejonders gegen Bären, Und fie nenten's Sienichenrechte!

"Menichenrechte! Menicjenrecjte! wer hat eud bamit belebnt?

Nimmer that es bie Natur, Dieje ifft nicht unnatürlich).

"Menj(jenrechte! Wer gab eud Dieje Privilegien?

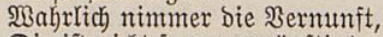
Die ift nicht fo unvernünftig!

,Menicjen, feid iłr etma befier, 2Ur's wie andre, weil gejotten IInd gebraten eure Speijen? $\mathfrak{B i r}$ verzehren rof bie unjern, 
Dodf Das Rejultat am Enbe Sit Daşelbe - nein, es abelt Nicjt bie 20bung; Der ift edel, Weldjer edel füblt und handelt.

, Menjacter, jeis ifr etwa befjer, Weil ifr \$siffenidjaft uno Rünite Mit Exfolg betreibt? Wir andre Sind nicht auf ben Ropf gefallent.

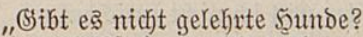
Itno auch Bferbe, welche rechnen Wie Rommerzienräte? Irommeln

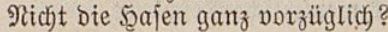

,5at fich nicht in 5obrofitatif Mandjer Biber ausgezeidynet? Und verbanft man nicjt Den Störchent Die Exfindung Der Rlyftiere?

„Schreiben Ejel nicht frritifen? Spielen $2(f f e n$ nidft Romb̈bie? Sitbt es eine gröpre Mimin, 2UI Batavia, Die Meerfał̨?

"Singen nicht bie Rachtigallen? S̃tt Der Freiligrath fein Dichter? 'Bet bejäng' Den \&öwen beffer

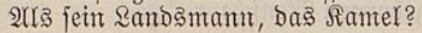

In ber Tanzfunft hab' idj jelber (ङ) jo meit gebrad)t mie Raumer In ber Schreibfunft - jd)reibt er befjer, 2(l’ ich) tanze, ich) Der Bär?

, Menicjen, marum jeib i hr befjer,

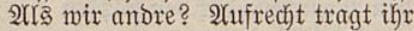
3war das 5aupt, jedoch im 5aupte Rriecken niedrig Die Siedanfen.

,Menichen, feid ifr etwa befler, 2̂t's mix andre, weil eu'r Felf Ślatt und gleip̈end? Diejen ßoxz̆ug Mü̈ït ifyr mit ben Schlangen teifert. 
,9) Menjchenvolf, z̧weibein'ge Schlangen, Ich) begreife wohl, warum ifs. 5ojen tragt! Mit frember 230 lie Dedft ifr eure Schlangennadtheit.

„Sinder! hütet eud vor jenen Unbehaarten Mifigeicböpfen! Meine Töb)ter! Iraut mux feinem Untier, welches ફ̧ojen trägt!"

Meiter will i(d) nicht bericjten, Wie ber $\mathfrak{B a ̈ r}$ in jeinem frechen (S)leich heitşโdmindel räjonnierte

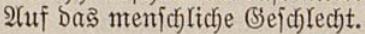

Denn am Enbe bin id felber 2(uch ein $\mathfrak{M e n j ( b ) , ~ u n d ~ w i e b e r b o l e n t ~}$ פBifl ich nimmer bie Sottifen, Die am Ende jebr beleio'gent.

Ja, ich) bin ein Menj(h, bin beffer NII bie andern Säugetiere; Die Intreffen Der SSeburt 'Sers' ich) nimmermehr ver(eugnen.

Into im Rampf' mit andern Beftien פुero' ich) immer treulich fämpfen

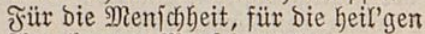
Ingebornen Menicjenrechte.

\section{Iaput VI.}

Doch es ift vielleicjt exjpriefilich F̈̈̈r Den Menjchen, ber Den höhern Niehítand bildet, bafi ex miffe, Was da unten räjonniert wiro.

$\Im a$, ba unten in ben büftern Jammeriphären ber (Sejelljwaft, Jn ben niedern Tiermeltichichten, Brïtet EITend, Stolz und Erroll. 
Maล naturgeichicbtlich immer, 2(rjo auch) gewohnteitsect)tlich, Seit Jahrtaujenden beftanden, Wird negiert mit frecher Sdnauze.

ßon Den 2YYten wiro Den Эungen Eingebrummt bie böje Эrrlebr', Die auf Erben bie Rultur Und Sumanität beoroht.

"Sinder!" - grommelt 2(tta Iroll, Und ex mälzt fitch hin und her 2(uf Dem teppic)lojen \&ager „Sintor, uns gefört bie 3ufunft!

,Dächte jeder Bär, und bäcjten 2ulle Tiere fo wie idf), Mit vereinten früften mürben ISir befämpfen bie Iyrannen.

"Sล verbände fict) Der (s)ber

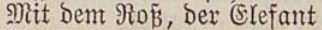

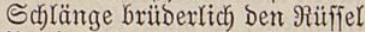
Utm Das Sorn bes madfern Ddjjen;

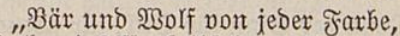
Sodf und 2lffe, felbit Der 5aje, Mirften ein'ge Beit genteinam, Und ber Sieg fömnt' una nicht fehlen.

"Einheit, Einheit ift bas erite Beitbedürfnis. Einzeln murben Sisir gefnechtet, Dod) verbunden übertölpeln wir Die 3wingherrn.

"Eintheit! Einkeit! und mix fiegen Und eอ ftürst das Regiment

Schnöben Monopola! Mit itiften (sin gerechtes 2lnimalreich).

"Sinumogejebs fei volle SSleichyeit 2rlfer Sintteafreaturen, Dhne Unteridied Des Stlaubent unt Desె J్lls und Des S'eruches. 
"Strenge (Sleidjheit! Jeber (5jel Sei befugt zum höbjten Staatşamt, Und Der Röme foll Dagegen Mit bem Sacf zux Mühle traben. "Tुas Den Sund betrifft, fo ift er Freilich ein ferviler Röter, शुeil Jahrtaujende findurch Э̧n Dex Menich wie'n Şund bejandelt;

"Doch in unferm Freiftaat geben Wir ifm wieder feine alten

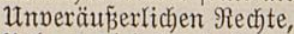
Und ex wird fitch bald veredeln.

"Iึa, jogar bie ฐuben follen Bolles Bürgerreç)t gentę̄en, Itnd gejebzlich gleichgejtellt jein 2rmen andern Säugetieren.

,Nux bas Tanzen auๆ Den Märften Sei Den Juden nicht gejtattet; Dies 2(mentement, ich mach' ę Эm Intreffe meiner Sunft.

,Denn Der Sinn für Stil, für ftrenge \$laftif Der Bemegung, fehlt Jenter $\Re$ affe, fie veroürben Den (Sefchmact bes \$ublifumts."

\section{ฐัaput VII.}

Düfter in Dex Düftern 5öhle Sodit im trauten אrrei 2itta Iroll, Der Menjdjenfeind, Und er brummt und fleticht bie Bähne:

,Nenichen, fdynippijche Ranaiffen! ¿ächelt mux! Bon eurem \&ächeln Wie von eurem jod) wiro endidi Int ber grofie Iag erlöjen! 
,Mich verleste ftets am meiften Jentę jauerjübe 3ucten UTm Das Maul - ganz unerträglich

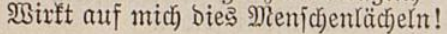

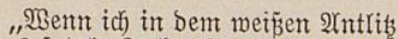
Dả fatale Bucten foraute, Drebten fich herum entrüjtet Mir im $\mathfrak{B a u c h e ~ b i e ~ S ’ e b a ̈ r m e . ~}$

,2Reit impertinenter noch,

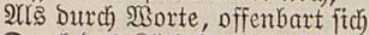
Durch Das \&ächeln eines Menjajen Seiner Seele tiefite Frechbeit.

IInmer lächeln fie! Sogar פ⿹丁 Dex 2Unftand einen tiefen Ernft exforbert, in ber ¿iebe Feierlichftem 2 ugenblicf!

„Iึmmer lächeln fie! Sie lächeln Selbjt im Ianzen. Sie entmeiben Solchermafien bieje fiunft, Die ein Rultủ bleiben follte.

"Iฐ̃ , Der $\mathfrak{L} a m_{z}$ in alten Beiten

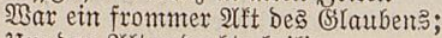
IIm Den Artar Drehte heilia

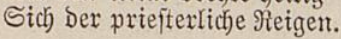
"2YTo vor ber Bundeslabe Ianzte weiland Rïnig Davio; Ianzen mar ein Siottesbienft, $\mathfrak{B a x}$ ein Beten mit Den Beinen! , 2XYjo bab' aucb) ich ben Tanz Einjt begriffen, wenn ich tanzte Uuf Den Märrften vor Dem ßolf, Das mix grofien Beifall zollte. ,Diejer Beifall, ich gefteh' e马े, Ihat mix manchmal mohl im Serzen; Dent Bemundrung jerbjt bem Jeinde

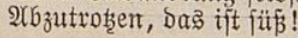




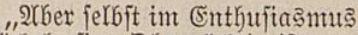
Sächeln fie. Dhnmä̈btig ift Selbit Die Tanzfunjt, fie zu befjern, Itno fie bleiben ftets frivol."

\section{æaนut VIII.}

Mancher tugenohafte Bürger Duftet ichlecht auf Erben, während Füritenfnecbte mit \&avendel SDer $2(m b r a$ parfümiert jind.

Jungfräuliche Seelen gibt eڤ̆, Die nach grüner Seife riechen, Itno Das \&after hat zumeilen Sich mit Rojenöl gemajchen.

Darum xümpfe nicht bie Naje, Teurer \&ejer, wenn bie 5̧̈̈le 2(tta Ixolla bich nicht erinnert 2) 2Trabiens Spezerein.

Meile mit mir in bem Dunjtfreis, Sn bem trüben Miñgeruche, \$ूo ber 5eld zu jeinem Sohne Wie aus einer $\mathfrak{B D}_{\text {olfe }}$ ipricht:

„Sind, mein Rind, Du meiner Senden Sัüngfter Sprößjling, leg Dein Einohr 2n Die Schnauze Des Erzeugers Und jaug ein mein ernites $\$$ ort!

,Süte bich vor Mienjchendenfart, Sie verbirbt bir Reib und Seele; Unter alfen Mienchen gift es Reinen ovidentficken Mienjcjen.

"Selbjt bie Deuticjen, einjt Die Befjern, Selbit bie Söhne Intiftions, Unire Bettern aus Der Itrzeit, Dieje gleichfalls find entartet. 
"Sind jebt glaubenlos und gottlos, Fres'gen gat ben 2rtheismus Rind, mein Rind, nimm bich in adjt ßox Dent Feuerbach ${ }^{1}$ und Bauer! ${ }^{2}$

,TSerbe mur fein 2rtheift, So ein Unbär obne (ङ)rfurcht \$or Dem Schöpfer - ja, ein Scjöpfer Sat erjofffen diejes şeltall!

I̊n ber Söhe Sonn' und Mono, 2(1ich) Die Sterne, (bie gejdumänzten (S)leichfalls wie bie ungefdymänzten)

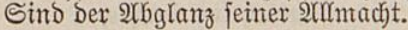

Iึn ber Tiefe, ฉand und Mieer,

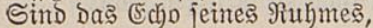
und jedmede Rreatur \$reijet jeine Serrlicbfeiten.

„Selbft bas fleinfte Silberläuscheri, Das im \$art bes greijen \$ilgers Ieil nimmt an ber Exdentwallfabrt, Singt Des En'gen sobgejang!

Droben in bem Sternenzelte,

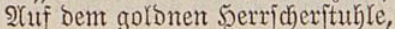
Weltregierend, majeftätijch, Sibt ein folofialer (5i引bär.

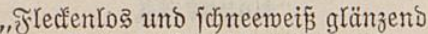

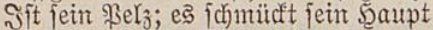
Eine Sirn' von Diamanten, Die burch alle Simmel Yeudtet.

"In Dem 2 Untlits Sarmonie Und Des Denfens ftumme Thaten;

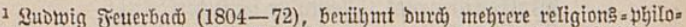
jopbijăe WBerfe, betonte Die Notmendigfeit einer bindologiidjen Erflärung Der religiöjen 2boritellungent unb befämpfte Den uniterblid)feitaglaubent.

a Brumo Bauer (geb. 1809) that fid ourd) freifinnige SBibelfritif herbor,

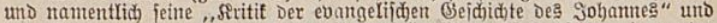

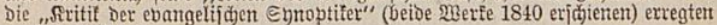
großెe 2lufieber. 
Mit Dem Scepter minft er mur, Und die Sphären flingen, fingen.

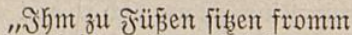
Bärrentheil'ge, Die auf Eroen Still gebuldet, in Den Taben ฐ̧res Märtyrertumes \$almen.

,'Miandjual fpringt ber eine auf, 2)uch Der andre, wie vom heil'gen Beift gemedt', und fieh! Da tanzen Sie Den feierlicbften Scodbtanz -

"5odatanz, wo Der Strahl Der Sinabe Das Ialent entbehrlich madjte, Itnd vor Seligfeit die Seele $\mathfrak{2}$ Uล Der 5̧aut zu fpringen jucht!

, Wherbe ich unmürb'ger Trom Einftens foldjen Seils teilkaftig? Into aus irdifach niedrer Irübjal übergehn ins Reich Dex Wonne?

"SBerd' ich felber, bimmerstrumfen, Droben in Dem Sternenzelte, Mit Der Silorie, mit ber ßalme Tanjen vor Dent Thron beב Şerm?"

\section{Taput IX.}

Wie bie fdarladjrote Bunge, Die ein fdrmarzer Frreiligräthicher Mohrenfürft verföhnens grimmig 2Utล Dem Düferm Maul hervoritredt:

2Ulp tritt Dex Nond auts Dunfelm \$ुolfentimmer. Fernther braujen Safferftürze, emig idjlaflos

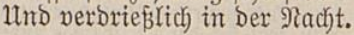

2rtta Troll fteht auf ber foppe Seines Sieblingsfelfens einfam, 
Einjam, uns ex heult bimunter

In Den Nacbtwint, in Den 2lbgrund:

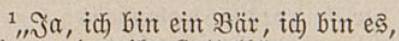
Bitr es, Den ifrr Bottelbär,

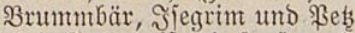

Into Ssott weis wie jonjt nod) nentet.

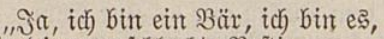
Bin Die ungejd)(ad) te Beftie,

Bin Das plumpe Irampeltier

Eures Şohnes, eures sächelns!

,Bin bie Bieljdjeib' eures Mikes, Bitn Das Angetüm, womit

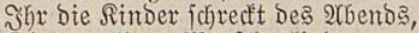
Die unart'gen Menjchentinder.

„Bin bas rohe Spottgebilde

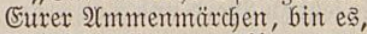
Itno idf ruf' es laut fimunter In bie jchnöbe Menjchentwelt.

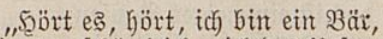

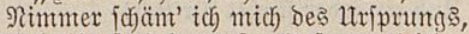
Itto bin ftol $\}_{3}$ barauf, als ftammt' ich 2Xb von Mojes Mendelsjohn!"

\section{Fiapit X.}

3wo (Sejtalten, wils und müruifd), Itnd auf allen vieren ruticjend, Brechen Babn fich burch Den bunflen Tannengumb um Mitternadjt.

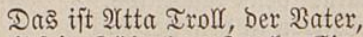
Itno jein Söhncjen, Jumfer Einohr.

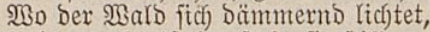
Bei bem Blutitein, ftehn fie ftille.

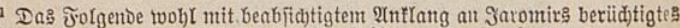
Bejtänonis, Daß̉ er ein Räuber fei (in Grillparjers "2ibnfrau"). 
Diejer Stein" - brummt 2Ytta Iroll "Ift Der 2Yrtar, wo Druiben

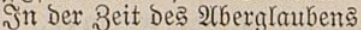

Menicjenopfer abgejchlacbtet.

,D Der fchauberhaften Greut!

Dent' ich bran, fträubt fich) Daङ Saar

2Uf Dem Rücf́en mix - Zur Ehre

Sinttes murde Bhut pergoffen!

"Ĩetst find freilich aufgeflärter

Dieje Menichen, uns fie töten

Nicjt einander mebr aus Eifer

Jür Die fimmlijcjen ฐntreflen; -

, Nein, nicht mehr Der fromme $\mathfrak{B a h n , ~}$ Nicht bie Sdjwärmerei, nicht Iolfkeit, Sondern (Eigennut und Selbitjucbt Treibt fie jeţt žu Nord und Iotjchlag.

, Nach Den Süitern biejer Erde Srreifen alle um bie 2 sette, Ind das ift ein ew'ges Raufen, Unto ein jeber ftieblt für fich)!

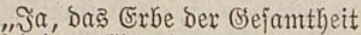
⿰习习̉ro Dem Einzelnen zux Beute, Und von Rechten Des Bejtizes Spricht ex Dann, von Eigentum!

"Eigentum! Recht Dę ßెefitfes! D Des Diebftahla! D Der Ritge! Solch Semific) won sift und Itnjinut Ronnte mux Der Nerrich exfinden.

, Reine Eigentümer icjuf Die Natux, Denn tajchenlos, Dhne Tajchen in Den $\mathfrak{B}$ elzen, Sommen wir zur Welt, wir alfe.

"Reinem von uns alfen murben 2lngeboren joldje Säct́djen

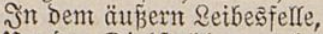
IIm Den Diebftabl zu verbergen. 
Nur ber Menjch, bas glatte \$sejen, Dả mit frember $\Re_{0}$ He fünttlich

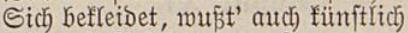
Sid) mit Iajchen zu verjorgen.

(Eine Ta[dje! Unnatürlidf) Sfit fie, wie Das Eigentum,

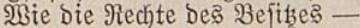
Tajkentiebe find bie Menjchen!

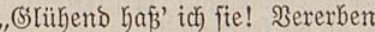
Will id bir, mein Sohn, Den Şafs. Sier auf Diejem 2urtar folljt bu

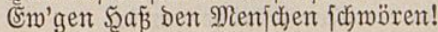

"Sei ber Iobfeins jener argen Interbrücfer, unver[öhnlich Bits ans Ende Deinter Iage, -

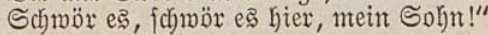

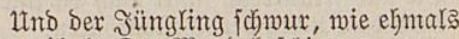
Samnibal. Der Mono bejcjien

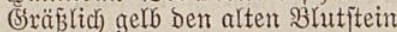
Uno die beiden Mifanthropen. - -

Später wollen wir berichten,

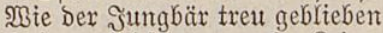
Seinem (Eidjofwur; unjre \&eier Feiert ihn int nädjten Epos.

शुaล Den 2(tta anbetrifft, So verlaffen wir ifn gleidffalla, Dod um jpäter ifn zut treffen Defto fichrer mit Der אugel.

Deine Itnterjudungsaften, Sodfverräter an Der Menj b heit 9) Tajeftät! find jebt gejchlofien; Miorgen wirb auf bich gefaljnot. 


\section{maput XI.}

Mie verid)lafne Bajaberen Schaun bie Berge, ftehen fröftelnd In Den meifien Rebelkemben, Die ber Morgenmind bewegt.

Dod) fie merben bald ermuntert ßon Dem Sonnengott, ex ftreift j̧nen ab bie letste Süfre Ind beftrahlt bie nadfte Sabönheit!

In Der Morgenfrübe mar $i d$ ) Mit ฉąfaro autsgezogen Iuf bie Bärenjago. Utm Mittag Ramen wix zum \$ont D'sfpagne.

So gekeiffen ift Die Brücfe, Die aus Frantreidy fülbrt nad Spanien,

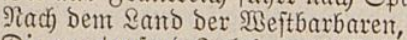
Die um taujend Jahr' zurücf jind.

Sind วurüdf um taujens ฐahre In moberner Seltgefittung Meine eignen Ditbarbaren Sind es nur um ein Jafrbundert. Bögerno, faft verzagt, verlief $i$ ) Den gemeibten Boben Franfreidjs, Diejes 刃aterlanos ber శrreikeit Int Der Frauen, Die idf liebe.

Mitten auf Dem ßont D' Sipagne Saṕ ein armter Spanier. Frend Saujchte aus bes Mantels söchern,

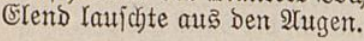

Eine alte Mandoline

Fineipte er mit magern Fingern; Schriller Mi șlaut, ber verböhneno

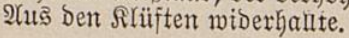


Manct)mal beugt' ex fich finunter Nach Dem 2(b grumb und er lacbte, Rlimperte nach her nod toller, Ind er jang Dabei bie \$3orte:

"Mitten Drin in meinem 5erzen Steht ein fleines güllones Iijd) hen,

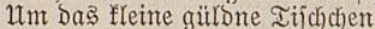
Stehn vier fleine güllone Stühldaen.

"श्2) Sileine Dämcjen, gülone \$feile Эm Ehignon; fie ipielen Rarten, 2łber Slara mux geminnt.

"Sie geminnt und läcbelt jacharfbaft, 2(ch), in meinem Serzen, Fllara, Wirit ou jedesmal geminnen, Denn Du haft ja alfe Irümpfe." -

Weiter mandelnd, zu mir jelber Sprach $i(d)$ : Sonderbar, Der SBabnjinn Sibt und fingt auf jener Brü̈ffe, Die aus Jrranfreich fübrt nad) Spanien.

Sift Der tolle ß̈uride' bas Sinnbilo

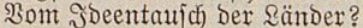

Doer ift ex jeines Bolfes Sinnverrüchtes Iitelblatt?

Gegen 2 (bend exit exreichten

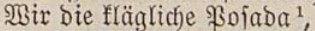
Mo bie Dllea \$otriba Dampfte in Der fdhmuts'gen Schüffel.

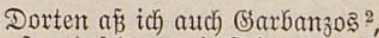
Srrofi und fchwer wie Flintenfugeln, Innerdaulich jelbit Dem Deutichen, Der mit אilöpen aufgemachjen.

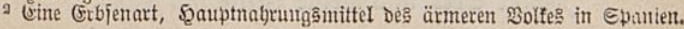




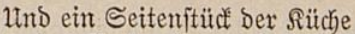
War Daక Bett. Şanz mit S̄njeften Wie gepfeffert - 2Ach! Die Manzen Sind des Mienjijen follimmite Feinde.

Schlimmer als ber 3orn von taujent Elefanten ift bie Feindichaft Einer einz'gen fleinen $\mathfrak{B} a n z e$, Die auf beinem \&ager friecht.

Muist Did̄ rubig beifjen Iaffen Das ijt fdylimm - Noch fchlimmer if Mुenn bu fie zerorücfift: Der Mifipouft Duält bich Dann bie ganze Nacht.

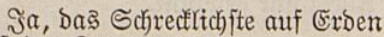
J̃it Der Rampf mit Ungeziefer, Dem Beftanf als Waffe Dient Dasె Duell mit einer \$anze!

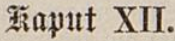

Wie fie formärmen, Die \$oeten, Selbft bie zahmen! und fie fingen Und fie fagen: Die Natur Sei ein grofier Tempel Sobttes;

Sei ein Tempel, beffen \$räcjte

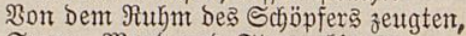
Sonne, Mono und Sterne bingen Dort als sampen in Der Ruppel.

Jึmmerhin, ifr guten Reute! Doch gefteht, in biejem Tempel Sind bie Treppen unbequem Rieberträchtig íchleç)te Ireppen!

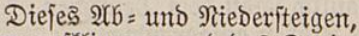
B̧ergauffimmen und Das Springen Über Blörfe, ę ermübet Meinte Seel' und meine Beine. 


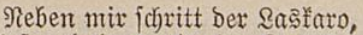
Şấ und lang wie einte Rerze!

siemals fpricht er, niemale lacht er, Er, Der tote Sohn ber Sere.

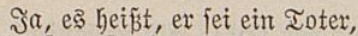
¿ärtgit veritorber, Doch Der Mutter, Der Urafa, 3auberfünfte Sielten jobeinbax ifn am Reben. -

Die vermünfditen Tempeltreppen! Dá̃ ich ftolperno in Den 2 (tbgrumb Nicht Den Şals gebrocjen mehrmals, Jit mix heut' noch unbegreiffich.

Wie bie Wafjeritürze freijchten! Wie ber Mind Die Tammen peitjdyte, Daßj fie Geulten! Plöslich plabten

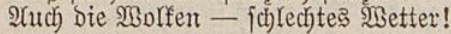

In ber ffeinen Fijcjerbütte, 2Un bem sac be Sobe fanden ISit ein Dboach und Frorellen; Dieje aber jabmediten föjtlich).

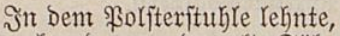
Siranf und grau, der alte Fährmant. Seine beiben fdjönen Nicjten, (Slleich) żwei Engeln, pflegten jeiner.

Didłe Engel, etwas flämifíd, SWie entiprungen aut Dem Rahmen Eines Ruben:s: gol'sne Rodfen, Rerngejunde, frare 2Uugen,

S5xübchen in Binnobermangen, Drin bie Schalffyeit heimlidy fichert, Innd bie Gifieder ftart und üppig, suft und Furcht zugletch) erregend.

Ђübjche, herzliche (Se)chöpfe, Die fich föftlich bisputierten: Meldjer Iranf Dem fiechen Dheim 2Sohl an bejten mumben mürbe? 
Şeinrid) Şeine.

Reicht Die eine ifm die Schale Mit gefochten Sinbenblüiten, Dringt die andre auf ibn ein

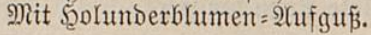

"Reins pon beiden will ich faufen", Rief Der Arte ungeduldig -

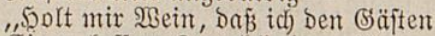
"Einen beffern Irunf fredenze!"

Db es wirflich Wein gemejen, Was icf tranf am Sac be S'obe, 23eī ich nicht. J̃n Braunichweig hätt' ich Wobl geglaubt, ę märe গجumme 1 .

Bon Dem befiten jdywarzen Bodfifell War ber Schlaud); er ftanf vorzüglich. Dody Der arre trant fo freubig, und ex mard gejund und beiter.

Er evählte uns die Thaten Der Banditen und Der Sdmuggler, Die ba Gaujen frei und franf In Den \$્\$yrenäenmäldern.

2(uch) von älteren (S̈ejc)idj)ten 2 Gubt' er viele, unter andern Uuch) Die Rämpfe Der (Siganten Mit Den Bären in Dex Borzeit.

J̃a, die Riejen und die Bären Stritten weiland um Die Serrichaft Diejer Berge, diejer Ihäler, (E)' die Menichen eingewanbert.

Bei Der Menjoben 2 (nfunft floken Yus bem sande fort die Riefen, Mie verblüfft; Denn menig Sirn Stedt in joldben gropien R̈̈pfen.

1 Starfes, Dunfelbraune: 5opfenbier von fiī̄em (Sejđumađ, Das in Braut=

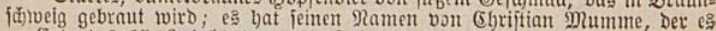
ju Ende Des 15. Jahrhunderts juerjt braute. 
2(uch) behauptet man: bie İ̈lper, Nris fie an bas Meer gelangtent Und gejehn, wie jich Der simmel In ber blauen flut gefpiegelt,

5ätten fie geglaubt, Das \$leer Sei ber Simmel, und fie ftürzten Sich) binein mit Sottvertraten; Seien jämtlich) Dort exjoffer.

$\mathfrak{W} a$ bie $B a ̈ r e n$ anbeträfe, So vertilge jest Dex Mienich Sie allmählich), jährlich f(brwände Jhre $3 a$ h in Dem Sebirge.

"So madjt einer" - iprach der 2rrte \$labs bem andern auf ber Erde. Sach Dem Untergang Der Menjiden fommt bie Serxichaft an Die Zwerge, ,22(n bie minzig flugen seutchen, Die im Schof der Berge haujen,

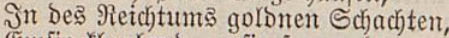
Emfig flaubent, emfig jammelnd.

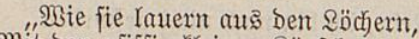
Mit Den pfiffig fleinen Röpfchen, Sab ich jelber oft im Monofdein, Und mir graute vor Der Zufunft!

, Nor Dex Gelomadjt jenter Snirpie! $\mathfrak{U}_{(c)}$, ich) für(b)te, unire Enfel

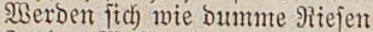
Эn Den \$afferfimmel f(üc)ten!"

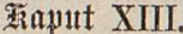

In Dem fochmarzen Felfenfeffel Rubt ber See, Das tiefe Maffer. Melandyolifich bleidje Sterne Schaun vom Simmer. Nacht und Stille. Seire. 2ttta Iroll. 
Racjt uns Stille. Ruberjaläge. M̉ie ein pläticherndes Śeheimnis

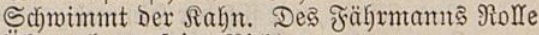
übernahmen feine Nichten.

Rubern flinf und froh. Sim Dunfeln seudjten mandbmal ifje ftämmig Ractiten $2(r m e$, fternbeglänzt, Und bie grofien blauen 2lugen.

Mix zur Seite fitst sasłaro, Wie gemöhnlich blä́ und ichmeigfam. Micb Durcbichautert ber (S'edanfe: Jift er wirflich nux ein Ioter?

Bin ich etwa felbft gejtorben, Itnd ich fififfe jetst bimunter Mit geipenitijajen Gejäbrten In Das falte Reich Dex Schatten?

Diejer See, ift ex des Styres Düftre Flut? Räpt ßrojerpine, Э̊n Exmangelung bes Eharon, Mich Durch ifre 3ofen holen?

Nein, id bin nod) nidjt geftorben und erloidjen - in ber Seele S'lüht mix noch) und jauchat und robert Die Yebend'ge Sebentsflamme.

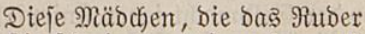
suitig ichwingen uns aud mand) mal

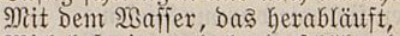
Midc) bejpriben, lacbeno, jääferno -

Dieje frijcjen, brallen Dirnen Sind fürmahr nicht geifterbafte Rammerfaben aus Der 5ölle, Ridyt bie 3ofen \$roferpinents!

Dẩ idf) ganz midy überzeuge ЭGree Dbermeltfichfeit, Ind dex eignen Rebensfülle 2(uch) thatjäd)(ich) mich verjidjre, 


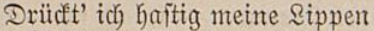

2(uf bie roten 2 angengrübchen, Ind idc) mad)te ben Nermunftichluf: Ja, idf) füfie, aljo (eb' ich!

2(ngelangt ans IIfer, füfit' ict) ఇoch einmal bie guten Mädchen; Nur in biejex Münze liȩ̄en Sie dą Fährgeld fich bezahlen.

\section{ฐaput XIV.}

2)us bem jonn'gen Siologrumb lachen Biolette Bergeßhöhen, Into am 2Ubhang flebt ein Dörfdjen, Wie ein fecfe马 \$ogelneft.

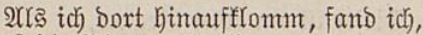
Dẩ bie 2rrten auggeflogen

Uno zurücf́geblieben nux

Junge Brut, Die noch nicht flügge.

Şübjche Bübchen, fleine Mäbdjen, Fait vermummt in idjarlachroten Doer weip̈en mollnen Rappen; Spielten Brautfahrt auf Dem Marftplab̨.

Sieß̃en fich im Spiel nicht ftören, Itnd ich jah, wie ber verliebte Mäujeprinz pathetij f) fniete Nor Der Raţenfaijerstodter.

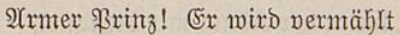
Mit Der Schönen. Märrifd zanft fite, Ind fie beifst ifn und fie frift ifn; Tote Maus, bas Spiel ift aus.

Faft ben ganzen Iag vermeilt' idf Bei ben findern, und wix idjwabten Sefr vertraut. Sie mollten miffien, Wer ich jei und was ich triebe? 
Rieben Jreunbe, - jprach $i$ ch - Deutid) Iand Seipit das sand, wo ich geboren; Bären gibt es bort in Mienge, Und icf wurbe Bärenjäger.

Manthem zog ich) Dort bą Frefl über feine SBärenohren.

शुohl mitunter mard id felber Starf gezauft von Bärentab̧en.

Doch mit \{chlechtgeledften Iölpeln నäglich mich) herumzubalgen In Der teuren Seimat, Deffen शुard ich endlich überoriiffitg.

und ich bin bierkergefommen, SBefires \$Beiomerf aufzujuchen; Mieine Rraft will idf weriudfen 2Yn Dem grofien 2 tta Iroll.

Diejer iīt ein ebler Gegner, Meiner mürdig. 2(d)! in Deuticblant Sab' ich mandben Rampf beftanben, N3o ich mich des Sieges ichämte. - -

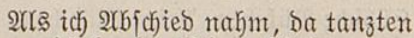
Itm mich her Die fleinen 2̧Bejen Fine Ronde, und fie jangen: "Śirofflino, Śirofflette!"

Rect und zierlich trat zulest જor mix Gin bie 2̂fferiüngite, Snirte zmeimal, oreimal, viermal, Und fie jang mit feiner Stimme:

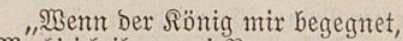
Mack' ich inm zwei Reverenzen, Und begegnet mix Die fiön'gin, Mach' ich Reverenzen orei.

"2YKex fommt mix gar Der Teufel In Den झुeg mit feinen Şörnern, Sinix' $i$ d) zmeimal, Dreimal, viermal Ssirofflino, Gsirofflette!" 
"Sitrofflino, Śtrofflette!"

Wieberfolt' Das Efor, und necfento

Mirbelte um meine Beinte

Sid) Der Ringeltanz und Singiang.

Währent id ins Thal hinabitieg, Scholl mix nach, verhallens lieblich; Эnmmerfort, wie ßogelzmitichert: "Sirofflino, Sirofflette!"

\section{ฐaput XV.}

Riejenhafte Feljenblödée, Mip̈geftaltet und verzerrt, S(baun mich an gleich Itngetümen, Die verfteinert, aub Der Inzzeit.

Seltjam! Sraue $\mathfrak{B o l f}_{\mathrm{f}}$ jajmeben Drüber hin, wie Doppelgänger; Sind ein blöbes fionterfet Jenter wilben Steinfiguren.

In ber Terne raj't ber Sturzbach, Und ber Wint Geult in Den F̈̈bren! (Ein (Seräuj(h), bas unerbittlich) Ind fatal wie bie ミerzmeiflung.

Scjaueriche Finjamfifeiten! Schmarze Doflenjcharen fitsen 2uf permittert morjchen Tannen, Flattern mit Den Lahmen Flügeln.

Reben mix geht Der Saßfaro,

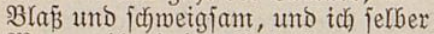
Mag mohl wie Der Wahnjtn auşehn, Den Der Yeis'ge Iod begleitet.

Einte bäß̈lich müjte Śegend. Riegt barauf ein Ituch)? Jd) glaube Bhut zu jeljen an ben \$urzeln Jenes જaum, Der ganz perfrüppelt. 
(5r bejdjattet eine Sütte, Die verjchämt fich) in Der (Erde 5alb veritedit; wie furchtjam flekend Schaut bich an das arme Strohbach).

Die Bemofner biejer Sütte Sind Eagoten ${ }^{1}$, überbleibjel Eines Stamms, Der tief im Dunfeln Sein zertretnes Dajein friftet.

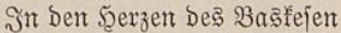

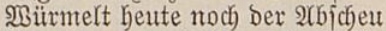
刃or Eagoten. Düftres Erbteil

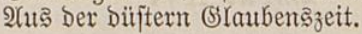

Эn Dem Dome วิ Bagnères Saujcht ein enges Sitterpförtchen; Diejes, jagte mir ber Rüiter, War Die Ihüre ber Eagoten.

Streng veriagt war ifnen ehmals Jeber anore ふitcheneingang, Und fie famen wie verftohlen Jn bą (Sotteshau gejulicjen.

Dort auf einem niebern Schemel Safis Der Eagot, einjam betent, Into gejondert, wie verpeitet, 刃on Der übrigen Siemeinto. -

2Hber die gemeihten Rerzen

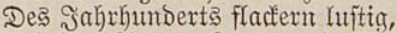
Ind Das Richt verjejeucht bie böjen Mittelalterlichen Schatten! -

Stehn blieb drauß̃en Der \&aร̈faro, Mährend ich in bes Eagoten

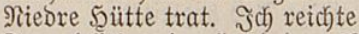
Freundich meine Şand Dem Brutoer.

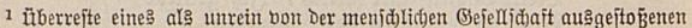
Utrolfs (Eagot von canis Gothus). Sie finton fid) in Sitofrantreid) und Spanien, baben gelblide 5aautjarbe und gelten für augjäbige Siretins. 


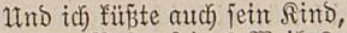
Das, am Bujen jeines গুeibes 2̂ngeflammert, gierig fautgte; Einer franfen Spinne glid) es̉.

\section{Taput XVI.}

Schaujt bu bieje ß̧ergesెgipfel 2(u Der Jern', jo ftrahlen fie, Mie geidnüudét mit Gold und \$urpur, Fürjtlich ftolz im Sonnenglanje.

श्ber in ber शähe fdymindet Dieje \$racht, uno wie bei andern Ĵroijchen Erhabenkeiten Iäuidjten bich Die Richteffefte.

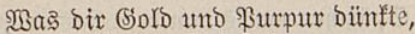
2(ch), Das ifit mux eitel Sdynee, (sitel Schnee, Der blöb und fläglida)

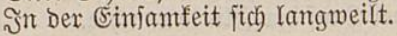

Dben in ber ఇähe fört' $i$ (d), MBie Der arme Sdnee gefniftert, Itno den fühllos falten 2 inden 2UI jein weî́es Elend flagte.

"D, mie Iangjam" - jeufat' ex - ,jchleidjen on Der SDe hier die Stumben! Diefe Stunden ohne Ende, Mite gefrorne Emigfeiten!

,$D$, ich) armer S(f)nee! D, wär' ich, Statt auf Dieje Bergeshöhen,

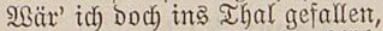
In bas ฐhal, wo Blumen brülen!

Singej dymolzen wär' ich) bann 2(r's ein Bächlein, und Des Dorfes Schönites Mäbchen wiljche lächelnd Эhr (Sefitht mit meiner 2 Eefle. 
nริa, id) wär' vielfeidjt gejchmonmen Bits ins Meer, wo ich) zur Perle Werben founte, um an Ende

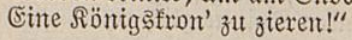

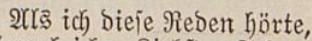
Sprach icf: „Riebfter Schnee, ich zweifle, Daj̃ in Ihale fold ein glänzeno Scfictjal bicb ermartet bätte.

"Iröjte Dich. Nur wen'ge unten Mुerben \$erlen, und bu fieleft Dort vielfeicft in einte \$fübe, Unto ein Dreç wärit bu geworben!" Mährento ich in jolcher झुeije

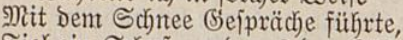

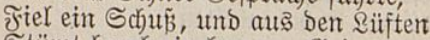
Stürzt Gerab ein brauter Śeier.

Späpidjen wax's von dem Iaßłaro,

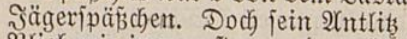
Slieb wie immer ftarr und exnthaft. Nur Der Sauf Dex Flinte raudste.

Finte Freder xif er joymeigend 2Ut Sie auf jeinten jpiben Filzhut, Unto ex j(britt bes \$Beges meiter.

Scbier unbeimlich war ber 2 (ntblick, Wie jein Schatten ntit Der Jeber

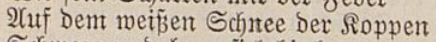
Sdjwarz und lang fich binbemegte.

\section{Finut XVII.}

Sift ein Thal gleich einer Saffe, Seifterhohlmeg ijt Der Nante; Schroffe Jeljen ragen jobmindicht (od) empor zu jeber Seite. 
Dort, ant jouarig jteilíten 2lbyang sugt ins Ihal, wie eine $\mathfrak{B a r t e ,}$ Dex Uraf́a fectes $ら$ äuslein; Dorthin folgt' $i c h$ Dem ¿asfaro.

Mit Der Mutter fielt er Rat In gebeimiter Beicjenjprache, Wiste Der 2(tta Iroll gelodit Und getötet werben fömme.

Dent mir batten jeine Fährte Siut eripütrt. Entrinten fonnt' ex Un's nicht meht. S'ezählt find beine Rebenstage, 2ttta Iroll!

Db bie 2ute, bie Itrata, Wirflich eine antagezeichnet Srofie Sere, mie die Reute כัn Den \$yrenä'n behaupten,

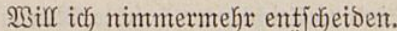

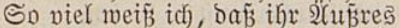
Sehr verdäcbtig. Sehr verdächtig Iriefent ifre roten 2 Utgen.

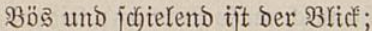
Und es heisht, Den armen Rühen, Die fie anblidft, trodfre plöblith In ber Euter alle Mitld).

Man verfichert gar, fie babe Streichelns mit ben bütren 5änDen Mandjes fette Sdymein getötet Utro jogar bie ftärfftert DChjen.

Soldberlei Berbrechent murde Sie żumeilen audf) verflagt Bei Dem Frtedentsridter. 2Uber Diejer wax ein Boltairianer,

(5in modernes flaches $23 e l t f i n s$, Dhne Iiefïnn, ohne Ślauben, Und bie Rläger wurben [feptij(t), Fajt verföhrtent, abgewiejen. 
Seinrid) Şeine.

Dffiziell treibt bie Utrafa

Sin Ṡejchäft, Dả jehr honett; Denn fite hanbelt mit Bergfträutern Unto mit ausgeftopften \$ogeln.

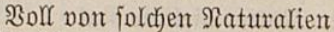
War bie Şütte. Sdjredt'lich rodjen Bilientraut uno Rucfudf̧blumen, Piffemurz und Iotenflieder.

Eine Rolleftion von Śeiem War vortrefflich) aufgeftellt, Mit Den ausgeftredten Flügeln Und ben ungebeuren Schnäbeln.

War's Der Duft Der tolfen Bflanzen, Der betäubend mix zu Ropf ftieg? शुanderjam mard mir zu Mute 32ei Dem 2Unblid Diejer ßögel.

Sind vielleicht vermünjchte Menichen, Die burch 3auberfunjt in biejent IInglüafiel'gen, ausgeftopften Љogelzuftand fich befinden.

Sebu mich an jo ftarr und reibent, und zugleich fo ungebuldig;

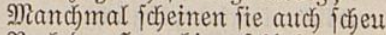
Nach) Der Şere Ginzufdielen.

Dieje aber, bie Itrafa, Rauert reben ifrem Sohne, Dem \&asfaro, am Ramine.

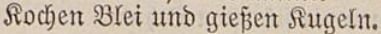

Sieß̃en jene Schichalätuger, Die Den 2itta Iroll getötet. ⿰豸勺⿱乛龰te bie Flammen Gaftig zudften uber bas Seficht Der Sere!

Sie bemegt bie Dünnen sippen Itnaufförlich, aber lautlos. Marmelt jie Den Dutbeniegen, Daí Der Rugelguí gedeifje? 
Manchmal fichert fie und nicht fie ЭGrem Solne. Alber diejer

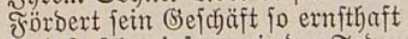
Ind jo jabeigjam wie Der Tod. -

Schwül bebrüct von Schauernifjen, (sing ich), freie auft zu jibjopfen, $2(n$ bas Jenjter, und ich icjaute Dort binab ins meite Thal.

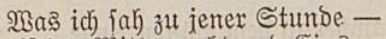
3wifd)en Mitternacht und (Eing 'Sुerd' ich trell und hübich berichten In Den folgenden Rapiteln.

\section{æaunt XVIII.}

Und es mar bie Zeit Des \$ুollmonds,

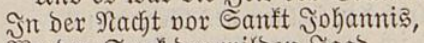
Wूo ber Spuf der willon JagD Utmzieht Durch Den Seifterhohlmeg.

2(นล Dem Fenfter von Urafas Serenneit fount' ich vortrefflich Das Seppenterheer betradjten, פूie es Durd die S'affe hinzog.

Şatte einen guten \$rab, Den Speftafel anzujchauen;

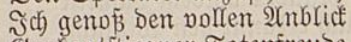
Sirabentitiegner IDtenfrellde.

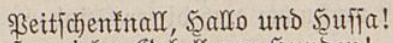

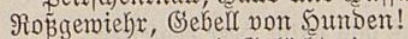
Jagohorntöne und (Selächter! Wie bas jaudjzend wiberhalfte!

¿ief voraus, gleicfjam alక গুortrab,

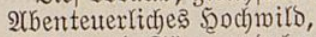
Şivich' uno Säue, molmeț; Seţent Ginterorein Die Meute. 
Эäger aus verichieonen 3onen Und aus gax verichiebnen Beiten;

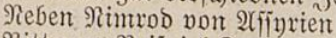
Ritt zum Beifpiel Rarl Der Zebnte.

Sodh auf weipen Rofjen jauften Sie Dahin. 3u ฮึนีืe folgten Die Sifeure ntit Der Roppel Und Die \$agen mit Den Fadfeln.

Mandjer in bem müjten Buge

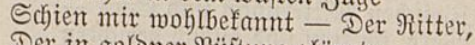
Der in goloner Rüitung glänzte,

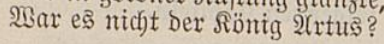

\section{Unt Şerr Dgier, Der Däne,} Intg er nicht ben fichifferno grünen Ringenpanzer, Da $\bar{s}$ er auşa Wie ein grofier Wetterfroj(d)?

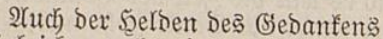
Sab ich mancken in bem 3uge,

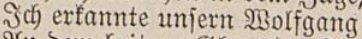
2tn Dem Keitern Slanz Der 2Tugen -

Denn, verbammt von Sengitenberg ${ }^{1}$, Rann er nicht im SFrabe ruben, Und mit Keisnijchem Selichter Seţt er fort Des Rebents Jagoluit.

2(n Des Nunbes holdem sächeln Ђab' $i(\bar{c}$ auch exfannt ben William, Den bie \$uritaner gleidgfalls Finft verffucht; auch Diejer Sünder

\section{Mǘ bas milde Seer begleitent} Racbts auf einem jichmarzen Rappen. গeben $\mathrm{ihm}$, пuf einem Ejel, Ritt ein Menich - Itno, beil'ger Şimmel!

1. Der theologijđje Eiferer Sengîtenberg $(1802-69)$, ßrofe jor in Berlin,

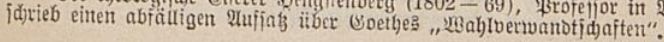


S(n Der matten Betermiene, Yu Dex frommen weifien Sdjlafmütş, In Der Seelenangit exfannt' id) Unjern alten Treund Franz 5orn ${ }^{1}$ !

Beil ex einit Das Weltfind Shafeppare Rommentiert, ntús jest Der \&̈rmite rach Dem Tode mit ifm reiten In Tumult ber willoen Jago!

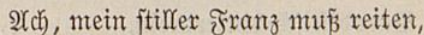
(5x, Der faum gemagt, zu geben, (5x, Der nux im Iheegejdynäbe Ind im Beten fitch berwegte!

NBerben nidyt die alten Jungfern, Die gejätjdyelt jeine Rube, Sich entieben, wenn fie bören,

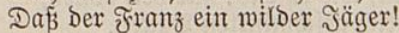

Wenn es mandimal in Salopp gebt, Schaut bex grofie William ipöttijch 2uf Den armen Rommentator, Der im sjelstrab inm nach folgt,

SSanz obnmächtig, feft fich frampend, Un Den Sattelfnopf DeS Srraudjens, Dod in Iobe, wie int Seben, Seinem 2utor treulid folgend.

Yuch Der Damen faf ich viele In Dem tollen Sieifterzuge, Sianz bejonders föbue Sinmphen, schlanfe, jugendlidbe seiber.

Rittlings fásen fie zu \$ferde, Mintfologifa fplittemadt; Dod) Die Saare fielen lodfigt sang herab, wie golone Miäntel.

1 Trang Sorn (1781-1837), als Didyter unbebeutend, rebieliger Sitterar =

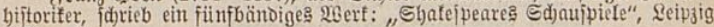
$1823-31$. 
Trugen אrränze auf Den Ђૃäuptern, und mit fed zuriäfgebognen, übermüt'gen \$ofituren

Schmangen fie belaubte Stäbe.

Rebert ifren jah idj ein'ge 3ugefnöpfte Ritterfräulein, Scjräg auf Dameniätteln fibent, und ben Jalfen auf Der Fauft.

\$̧ Sarobififich Ginterorein, 2(uf Scjinomähren, magern Rleppern, Ritt ein Trof́ von fomtöbiantij(b) Uufgepubsten \$ָeibsperjonen,

Deven 2 (ntrib reizens lieblich), Ther aud ein bifschen frech.

Schrien, wie rajend, mit ben vollen, sieberlich gejdminften \$adfent.

Mie bas jubelno miderkalnte! ฐaģohorntönte und S’elächter!

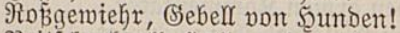
\$eiticjentralf, 5allo uno 5̧uffa!

\section{Iaput XIX.}

2Uber als Der Sdjönheit Rleeblatt Ragten in Des Buges Mitten Drei Sieftalten - Nie vergeff' $i c$ h Dieje Golden Jrauenbilber.

Reidjt exfennbar mar bie eine Un Dem 5albmond auf bem Şaupte; Stolz, wie eine reine Bildjäul',

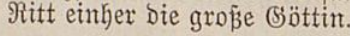

Şochgeichürzte Tunifa, Bruft und 5üfte halb bebecfend. 
Fracfellicbt und Monojichein fpielten siijtern um bie meipen SSlieder.

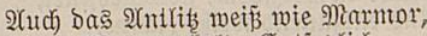
Itnd wie Marmor falt. (Entjeblich War bie Starrbeit und bie Bläffe Diejer ftrengen eblen Büge.

Dod) in ifrem jowwarzen 2 tuge Soberte ein arauenhaftes Und untheimlich füßses Fener, Seelenblentent und verzebrent.

WSie verändert ift Diana, Die, im übermut Der Reujch)heit, Einft Den 2lftänn verbirichte Int Den Şumben preisgegeben!

Büīt fie jeb̨t für bieje Sünde In galantejter Sejellic)aft?

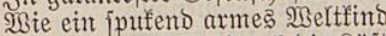
F̈ährt fie näcbtlich) Durch Die süfte.

Spät zwar, aber befto ftärffer Sift ermadyt in ihr bie \$Bollupt, Into es brennt in ifren 2Uugen Wie ein wahrer Şöllenbrant.

Die verlorme Beit berent fie, WSo Die Männer ichönter waren, Inno bie Duantität erjest ibr Jesct vielfeicht Die Dualität.

Reben ifr ritt eine Schöne, Deven Büge nicht io griechij Streng gemefien, Dod fite ftrahlten 刃on Des Eeltenjtammes 2)

Diejes mar bie Fee 2 trumbe, Die idf Ieicht erfenten fonnte 2(r Der Sübe ihres Sächeln' Into am herzlich tollen 2acjen! 


\section{Secintid) Speitte.}

Ein (Sefitcht, gejund und rofitg, W3ie gemalt won Mleifter Srewze 9) cund in Serzform, itets geöffnet, Ind entzüdtend meiffe 3ähne.

Irug ein flatterno blaues Rachtffeid,

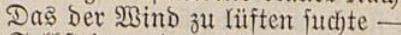
Selbit in meinen beften Iräumen Sab ich nimmer joldje Schultern!

Wenig feglte und idj fprang 2us dem Jenfter, fie zu fuiffen! Diejes wär' mir fchlecht befommen, Denn Den Şals hätt' ich gebrocber.

2(c) ! fie hätte mur gelacht, SBenn ich unten in bem 2tbgrund

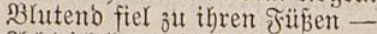
$($ (d) ! ich fente jolches sachen!

Und das dritte Frauen bilo, Das Dein Serz fo tief bemegte, פूar es eine Ienfelinne, Wie Die andern zwo Sieftalten?

Db's ein Teufel ober Engel, গูei[ $i$ ch nicht. Senau bei Meibern Weif man niemals, wo ber Engel 2(ufbört und ber Teufel anfüngt.

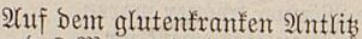
Zag bes Morgenlandes Zauber, Uluch die Rleiber mahnten foftbar Un Scjeberezadens Miärchen.

Sanfte Sippen, wie Srenaten, Fin gebognes Rifiennï̈s)en, Und bie Ślieber id)lanf unD füblig Wie Die \$alme Der Daje.

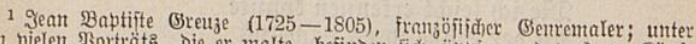
ben vielen \$orträts, Die er malte, befinden itd) iippige und pactende fiöpie und Bruitbilder von jungen Jrauen uno Mä̉d jen 
Sehnte hoch auf meinem Belter, Deffen Soldzaum von zmei Mobren

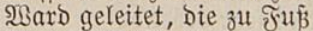

2Un Der Fürjtin Seite trabten.

Wirffich eine F̈urftin war fie,

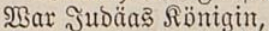

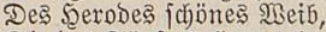
Die Des Täufers 5aupt begehrt bat.

Diejer Blutjouls halber ward jie 2(uch) vermaledeit; als Nachtipuf Muf fie bis zum jüngften Iage Reiten mit ber wilden Jago.

In Den Sänden trägt fie immer Sene Schüffel mit Dem Saupte Des Johannes, und fie füfít es; ฐa, fie füpt Das Şaupt mit Inbrunft.

Denn fie liebte einjt Johannem Sn Der Bibel fteht es nicht, Dody im 刃olfe lebt die Sage ßon Serobias' blut'ger Siebe -

2(nders mär' ja unerfrärlich Das Belüifte jenter Dame Wiro ein $20 e$ ba Da 5aupt begehrent Eines Manns, Den fie nidjt liebt?

2ुax vielleidyt ein bişchen böje

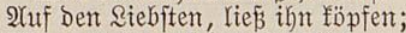
2tber als fie auf Der Sdüinflel Das geliebte Scaupt erblicte,

Weinte fie und ward verrültt, Itnd fie ftarb in Riebesెmaknitnn. (Riebesmahninn! \$leonasmus! Liebe ift $j a$ jabn ein $\mathfrak{B a b n j t n n ! ) ~}$

Nächtlich auferjteheno trägt fie, Wie gejagt, bas blut'ge Scaupt In ber Sano, auf ifrer Jagofahrt Doch mit toller $\mathfrak{B S}_{\mathrm{B}}$ iberlaune 
Schleubert jie bas Saupt zumeilen Durch bie Rüfte, findif la lackend, Ind fie fängt es fehr behende Wieber auf, wie einen Spielball.

ẩ fie mir vorüberritt, Schaute fie mich an und nicfite So fofett zugleich und famadjtent, Daf́s mein tiefítes Şerz erbebte.

Dreimal auf uno nieber mogeno Fubr ber $3 u g$ vorbei, und dreimal Эm 刃orïberreiten grüp̈te Mich Das liebliche šejpenjt.

Irs ber Bug bereits exblichen Itnd verffungen bas S'etümmel, Soberte mix im SSebinne Ĵmmer fort Der holde Siruf.

Und bie ganze Nacht hindurd

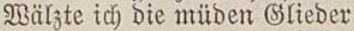
YUf Der Streu - (Denn Feberbetten (Sab's nicht in Itrafas ફüitte) -

Itno ich fann: was mag bebeuten Das geheimnisvolle Ridfen? \$ुarum haft Dut mich fo zärtlich 2(ngejebn, Serobias?

\section{Faput XX.}

Eonnenaufgang. Solone \$feile 〔chiefen nach Den meifien Nebeln, Die fich röten, wie vermundet, Itno in SIlanz uno Sidjt zerrinmen.

(Endich) ift ber Sieg exfochten, Und Dex Tag, Der Iriumphator, Iritt in ftrahlent voller slorie ?huf Den Racten Des Sebirges. 
Der S'evögel Yaute Sippichaft 3witjchert in verborgnen Nejtern, Und ein Rräuterourt erhebt fich,

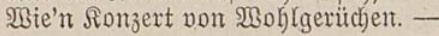

In ber erften Ncorgenfrübe Waren wir in Ifal geftiegen, Und bermeilen ber \&asfaro Seines Bären Spux verfolgte,

Sudyte id die Beit zu töten Mit (Sebanfen. Doch Das Denfen Machte mich ant Enbe mübe Und jogar ein bifichen traurig.

(Enolid) müd' und traurig fanf ich Nieber auf bie meiche Moosbant, Intex jener grofien Ej che, Wo bie fleine Duelle flof́,

Die mit wunberlichem ßä̈tjchern 2rjo wumberlich betförte Mein S'emüt, Das Die Śebanfen Und Das Denfen mir vergingen.

(ङङ exgriff mich milde Sehnjucht

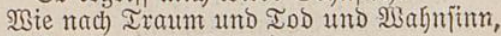
Und nach jenen Reiterintmen, Die ich jah im S̈eijterbeerzug.

D, ifr Golden Nachtgefichte, Die Das Morgenrot verjajeuchte, Sagt, wohin jeib ifre entfloken? Sagt, wo haujet iht am Tage?

Inter alten Iempeltrümmern,

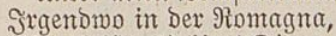
(Alijo beis̄t es) birgt Diana Sich) vor (Ehrijti Iagesherrichaft.

ఇux in mitternäcbt'gem Dunfel Wagt fie es herworzutreten, Itnd fie freut fich Dann Des \$Bseidwerts Mit ben Geionitichen Gsejpielen. 
2(uch) Die fchöne Fee 2Ybunde Fürchtet fich vor शazarenern, Und ben $\mathfrak{T} a g$ bintourch vermeilt fie Эn bem fitchern 2(valun.

Diejes Eiland liegt verborgen Ferne, in Dem fitllen Meere Der Romantif́, mux erreicbbar 2Uf Deక శabelrofles Frügeln.

Riemals anfert dort bie Sorge, Niemale landet Dort ein Dampfichiff Mit nengierigen \$Giliftern, Iabafspfeifen in Den Mäulern.

Niemale bringt oorthin bas blöbe Dumpf langweil'ge Ślocfenläuten,

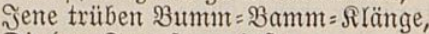
Die ben Feen jo verhajit.

Dort, in ungeftörtem Frobfinn, und in ew'ger Jugend blübend, Refitiert Die Geitre Dame, Injire blonde Frau Stbunde.

sachend geht fie bort ipazieren Inter hohen Sommenblumen, sit bem fojenden S'efolge Weltentrüdfter \$alabine.

थtber Du, 5erobias, Sag, wo bijt du? - 2(d), ich weifis es, Du bift tot und liegít begraben Bei der Staot Jerujcholayim!

Starren Reichenj(blaf am Iage Schläfft bu in Dem Marmorjarge! Doch um Mitternacht ermedit bich \$eitichenfnall, Şallo uno Suufja!

Itnd Du folgit Dem willoen Sceerzug Mit Dianen und 2tbunben, Mit ben Keitern Jagogenofien, Denen Sireuz und Dual verhajit ift! 
Whelche föptliche (sejellichaft! Rönnt' ich) näcbtlich) mit euć) jagen Durch) bie Wälder! Dix zux Seite Ritt' ich ftets, Serodias!

Denn id liebe bich am meif́ten! Miehr als jene Ëriechengöttin, Mehx als jene free bes Norbent, Sieb' ich Dich, Dut tote J̈̈̈bin!

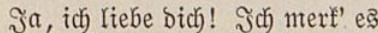
2(n Dem Bittern meiner Seele. siebe mich und jei mein siebchen, Scjöne: \$eib, 5erodią!

Siebe mid und jei mein siebchen! Schleutre fort Den blut'gen Dummfopf Samt Der Schüfjel, uno gentef̧e Sdymacthaft beffere (Seridjte.

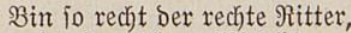
Den bu braucbjt - Mitc fümmert's wenig, Dafis bu tot und gav verbammt bijt Sabe feine Norurteile -

5apert's Doch mit meiner eignen Seligfeit, und ob ich jerber Noch Dem Reben angeböre, Daran zmeifle ich zumeilen!

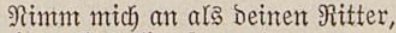
Deinen (Eavalier=jervente; Werbe Deinen Mantel tragen Unt audi) alle beine saunen.

Tebe Racht, an beiner Seite, Reit' ich mit Dem wilben seere, Und wix fojen und wir lacken über meine tolfen Reden.

Werbe bir bie Beit verfürzen

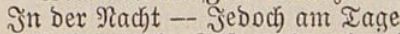
Schwindet jebe \&ujt, und meinend Sits' ich bant auf Deinem Ssrabe. 
ฐa, am Tage fits' ich meinend গuf Dem Schutt Der Rönigggrüfte, Yuf Dem Srabe Der Sieliebten, Bei Der Stabt Jerujablanim.

rrte Juben, bie vorbeigehn, Slauben bann gemi $i$, ich traure Db Dem Untergang Des Tempels und ber Stadt Jevujcholanim.

\section{æaput XXI.}

Yrgonauten obne Sdhiff, Die zu ₹rús gebn im SSebirge,

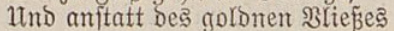
Nux ein Bärenfell exzielen -

Y(d)! wix find nux arme Teufel, Selden oon modernem Bujdnitt, Und fein flaffifdier \$ot 2Bito ans im Siejang verem'gen!

Und wix Gaben Doch erlitten

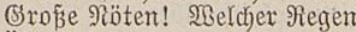
ïberfiel uns auf Der Roppe, 230 fein $B$ aum uno fein Fiafer!

Wolfenbutd)! (Das Brudjband plabte.) Rübelmeis ftürzt' es herunter! Jajon ward gewij auf Rolchis

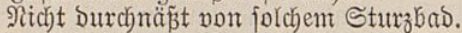

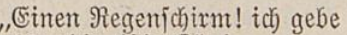
Sectiundoretîtg fïnige Jebt für einen Regenjchirm!"

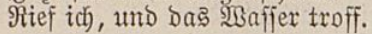

Sterbensmübe, fehr verorię̧lid

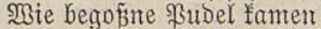

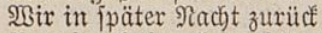
গach Der Goben Sexenhütte. 
Dort am Yidhten freuerherbe

Saís Urafa und fie fämmte

shren grofien, diden Mops.

Diejem gab fie janell Den ¿aufpaí,

Um mit uns fich zu bej chäft'gen.

Sie bereitete mein Sager,

¿ïfte mir Die siparbiflen,

Diejes unbequente ₹̛̣̋zeng,

Salf mix beim Entfleiben, zog mix

खuch Die sojen aus; fie flebten

Sir am Beine, eng und treu,

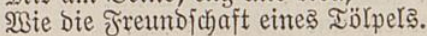

,Einen Schlafrodf! Sechsundoreifig

Rönige für einen trodfnen

Schlafrod́l" vief ich), und es bampfte

Mix Das nafje Semb am seibe.

Fröftelnd, zäfneflappernd ftanto ich

Fine SBeile an Dem Serbe.

शूie betäubt vom Feuer fanf id

Enblich nieber auf die Streu.

Ronnt' nidbt joblafen. Bringelno jobaut' idj Sach . Dex Sex', Die am Ramin jaf

IInd Den Dberleib Des Solnes,

Den fie ebenfalls entfleidet,

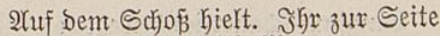
2(ufredst ftand dex Didfe Mops

Und in jeinen 30 rDerpfoten

Sielt er fehr gejdidft ein Iöpfdyent.

צ(us Dem Iöpfchen nahm Uraf́a Rotes Fett, beftrich Damit

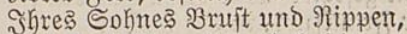
タieb fie haftig, zitterno hajtig.

Utno dermeil fie rieb und farbste,

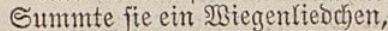
গiäjelnd fein; bazmijđjen feltjam Anifterten Des Serdes J Iammen. 
Wie ein Reidfnam, gelb und fnöbjem, Sag ber Sohn im Schoi ber Mutter; Iobestraurig, meit geöffnet Starren jeine bleichen 2 (ugen.

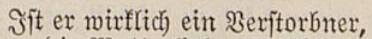
Dem bie Mutterliebe nächtlich) Mit ber ftärffiten Şerenjalke Ein verzaubert \&eben einreibt? -

SHunderlicher Fieberbalbid)laf! Wo bie Slieber bleiern mübe গুgie gebunden, und bie Sinne überreizt und gräßj(ich) mad)!

Wie Der Rräuterouft im Bimmer Mich) gepeinigt! S(f)merzlid) grüberno Sant ich nad), wo idj Dergleidjen Schon gerochen? Sann vergebens.

Wie ber $\mathfrak{B i n}_{\text {zug in }}$ Ramine Micb) geängitigt! Rlang wie 2 (d) zen Bon getrodnet armen Seelen Scjienen mohlbefannte Stimmen.

Doch) zumeift waro ich gequält જon Den ausgeftopften ßögeln, Die, auf einem Brett, 子u Б̋̈upten Neben meinem Rager ftanben.

ฉangiam ichauerlich bemegten Sie bie Flügel, unt jie beugten Sich) zu mix herab mit langen Schnäbeln, bie wie Menjchennajen.

2(d)! wo hab' idf joldje शajen Schon gejehn? \$ָar es zu Samburg Doer Franffurt, in ber Saflïe? Qualvoll bämmerno bie Erinnung!

(snolich) übermannte gänzlich Mich) Der Schlaf, und an bie Stelle Wachender Fyantasmen trat Fin gejunder, fefter Iraunt. 


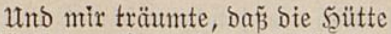

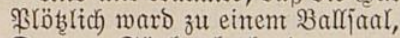
Der von Säulen hodjgetragen Itno exhellt von Sirandolen.

\section{Unfitchtbare Mufiffanten}

Spielten aus Robert le Diable

Die verruchten Nonnentänze;

Sintg bort ganz allein ipazieren.

(Enolich aber öffnen fitch

Meit bie \$forten, und es fommen, ¿angjam feierlicjen Schrittes,

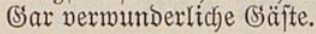

sauter Bären uns Siejpenfter! 2(ufrect)t wandelnd, füfrt ein jeder 刃ुon Den Bären ein Sejpentt,

Das vermummt im weipen (Śrabtud).

Soldjermeis gepaart, begannen Sie zu malzen auf uns nieber Durct) Den Saal. Ruriojer 2tnblide! 3um Erjachredien und zum \&acjen!

Denn ben plumpen ß̧ären maxd eకె Serzlich fauter, Schritt zu Galten श)it Den weifien Ruftgebilben, Die fich mirbelno leidjt bemegten.

Unerbittlich fortgeriffen Murben jene armen Beftien, Into ihr Schnaufen überoröbnte

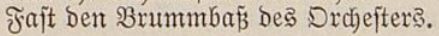

Mandymal malzten fich bie \$aare YUf Den Reib, und Dem Śejpenfte, Das ifn anjtieñ, gab ber $3 a ̈ r$ Fin'ge Iritte in Den Sintern.

Mandbmal audb, im Tanzgetümmel, Riti Der $\Re$ är bą Seichenlafen

刃on Dem Saupt DeS Tanjgenoflen;

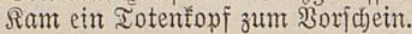


Snorid) aber jaudbsten idjmetterno Die Irompeten uns Die 3 imbeln, Und es onnerten die \$aufen, Into es fam die Saloppabe.

Dieje träumt' idy nidgt zu Enbe Denn ein ungejd lad)ter $\mathfrak{B} a ̈$ r Irat mix auf Die Sühneraugen, Dẫ ich aufichrie und ermadyte.

\section{æaput XXII.}

\$hb̈bus, in Der Sonnendroidje, \$ुeitichte jeine Jlammentofie Und er hatte fabn zur Sälfte Seine Simmelsfabrt vollendet -

शुäbxend ich im Schlafe lag Ints von SBären und (S)ipenjtern, Die fich munberlich) umichlangen, Tolle গrabesfen! träumte.

Mittag mar's, ars ich exmadjte, Itnd ids fand midy ganz allein. Meine Birtin und $\Omega$ asfaro Singen auf Die Jago jabn frübe.

ฐn Der Süitte blieb zurüc Nux Dex Mops. IIm Feuerherde Stand er aufrecht por Dem Reffel, Jn Den ßfoten einen söffel.

Scbien vortrefflich abgeridjtet, Sgenn die Suppe ïberfochte, Schnell Darin herumzurübren Und Die B̧lajen abzujchäumen.

Uber bin ich jelbjt behert? Doer lobert mir im Ropfe Roch Das Fieber? Meinen Dhren (S) Yaub' ich faum - es ipridyt Der Mopङ! 
ฐa, ex \{pricht, und zmar gemütlich Schrö̈bijc) ift bie Mundart; träumtento, Mie verloren in Ssedanfen, Spridgt er folgendergeftalt:

,D, i if armer S(f)mabendichter! In Der Frembe muí ich traurig 2)( Unto Den Бerenfefjel hüten!

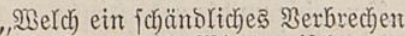
Sft' Die Zauberei! Wie tragif(

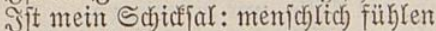
In Der Şülle eines Şundes!

,'23är' ich Doch Daheim gebrieben, Bei Den trauten Schulgenoffen! Das find feine Serenmeifter, Sie bezaubern feinen Menjajen.

, , Bei fiarl Maner, bei ben jüfen Selbveiglein des গaterlandes, SBet Den frommen Meţeljuppen!

Seute fterb' $i c$ fajt vor Seimmeh -

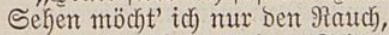
Der emporiteigt aut Dem Scjornitein, Wenn man Rubeln focht in Stuffert!"

2rrs ich dies vernalym, ergriff mich Tiefe Rübrung; von Dem Rager Sprang ich auf, an bas Ramin Seţt' ich mich, und pprach mitleidig:

„(5) ler Sänger, wie gerietejt Dü in bieje Serenhütte?

Itno marum hat man fo graujam Did) in einen Scund vermandert?"

Jener aber rief mit Freube: ,भIfo find Sie fein Jranzofe? Sind ein Deuticher, und verftanden Meinen ftiflen Monolog? 


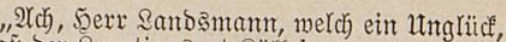

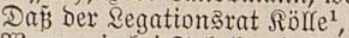
গুSenn mix bei Iabaf und Bié Jn Der Rneipe Dißfurierten, "İmmer auf Den Sał zurüdfam, Man ermürbe nur burch Reijen Jene Billoung, Die er jelber Uน' Der Frembe mitgebracht! "IIm mir mun bie roke Rurute ßon Den Beinen abzulaufen, Und, wie Rölle, mir bie feinern Weltmannşitten anzuja)leifen:

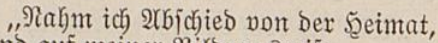
Utnd auf meiner Bildungereije Ram icb nach) Den \$yrenäen, Nach Der 5ütte Der Urafa.

"Bracht' ifr ein Empfehlungşăreiben ßon Jutimu Rerner; Dachte

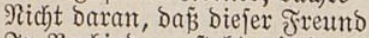
In হerbindung fteht mit Seren ${ }^{2}$.

"Irreunolich nafm mich auf 1 rafa, Dod) es mudbs, zu meinem Schrecfen, Dieje Freunolidffeit, ausartend Enolich gar in Sinnenbrunft.

"Ĩa, eכ flacterte bie Inzucbt Scheufilich auf im melfen Bujen Diejer lafterbaften Bettel, Und fite wollte mich verfübren. , Doch ich flebte: 2 (ch, entichuls'gen Sie, Mabame! bin fein frivoler

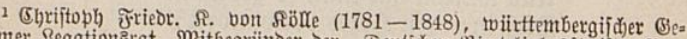

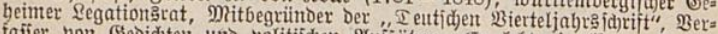

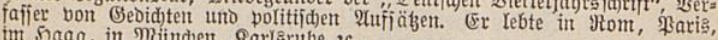
im Seag, in Miind)en, Rarlsrube 2 .

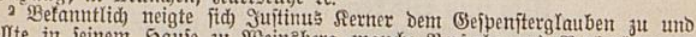
ftellte in feinem Sgauje ju Weingberg manthe Beriude und Beobadtungen

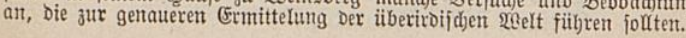


Sibetheaner, ich gehöre

3ె ber Didbteridule Scbuabens.

"Sittlicbfeit ift limire Nuje, Und fie trägt vom bicfiten Reber

Itnterfojen - $2(d)$ ! vergreifen Sie fitch nicht an meiner Iugend!

"2Anore Dichter haben Beift, 2(nore \$hantafte, und anore Reibenidjaft, jeboch Die Iugend Saben wir, die Schmabenoidyter.

"Das̉ ift unjer einz'ges Grut! Rauben Sie mix nicht Den fittrich Religiöjen Bettelmanter, Meldher meine $\mathfrak{B l o ̈ p e}$ Dedft!

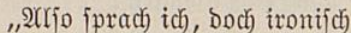
¿ächelte bas $930 i b$, und lächelno Rahm fie eine Miftelgerte Inno berüGrt' Damit mein Şaupt.

Iรch) empfand alsbald ein faltes

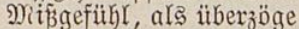

Eine S̈änjehaut Die Sitieder.

Doch) Die Saut von einer Sant

, $13 a r$ es nicht, es war vielme hx Eines Sundes Fell - Seit jener In heilftund' bin ich) vermanbelt, Mie Sie jehn, in einen Mops!"

2(rmer Sdjelm! গুor Iauter Sd)luthjen Ronnte er nicht weiter iprecken, Inno er weinte fo beträglich, Daj er faft zerflofis in Ihrämen.

"Sören Sie", fprach id mit "אann ich etwa von dem Sundşfell Sie befrein, und Sie ber Didftfunft Itno ber Mienjchbeit miedergeben?" 
Sener aber hub wie troftros und verzweiflungşoll bie \$foten Эn bie 5öhe, und mit Senfzen Und mit Stöbnen iprach ex endicfj:

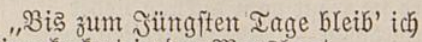
Eingeferfert in Der Mopshaut, Wenn nicht einer Jutrgfrau Sirofint Mich erlöjt aus ber Bermünjchung.

ฐ̃a, mux eine reine ฐungfrau, Die nod) feinen Mann beriibrt bat, Ints bie folgente Bebingung Treu exfürt, fann mich erlöjen:

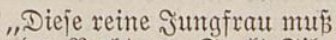
In Der Nacht von Sanft Silvefter

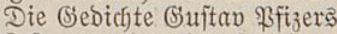
sejen - ohne einzujhlafen!

, 3 lieb fie mach bei ber Reftüre, Schlofi fie nicht bie feujchen 2(ugen Dann bin icf entzaubert, menjajlict) 2(tm' ich) auf, icb) bin entmopft!"

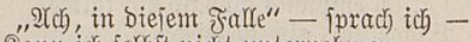
fiann idj) felbft nidjt unternebmen Das Erlöjungsmerf; Denn erftent Bin $i$ (d) feinte reine J̃ungfrau, "Itnd im ftande wär' ich zmeitens Noch viel wen'ger, Die SSedichte Siuftav Pfizers je zu Yejen, Dhne Dabei einzujalafen."

\section{æạut XXIII.}

2us Dem Spuf Der Sexenmirtjchaft Steigen wir ins Ihal herunter; Unjre füize fafien wieber Boben in Dem \$ofitiven. 
Frort, Bejpeniter! Nadjtgefichte! Suftgebilde! ₹̛̃eberträume! פ⿹\zh13一 beichäft'gen unt vernünftig Mieber mit Dem 2̂tta Iroll.

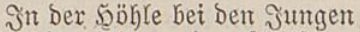
Rieat ber 2Ulte, und ex id) läft Mit Dem Stjnarchen bes (Serechten; (Enolich macht ex gähnend auf.

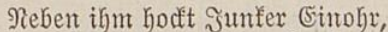
Itnd ex fratst fich an Dem Ropfe IBie ein Didfter, Der ben Reim jucjt; Ifuch ffandiert ex an ben Iaben.

Sেleidfafles an bes Baters Seite Siegen träumend auf Dem Rücf́en, Inthuldorein, vierfüв̈'ge হilien, Utta Trollg geliebte Töbter.

Melche zärtliche Siedanfen Schmadjten in ber Blütenjeele Diejer meif̧en Bärenjungfraun? Ihränenfeucbt find ibre sBlicfe.

Sanz bejonders jcheint bie jüngfte Tiefbemegt. In ifrem 5erzen

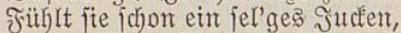
Uhndet fie die Macht Siupidos.

ฐa, ber Bfeil bes fleimen Ssottę Sit ifr burch Den $\mathfrak{B e l} z$ gebrungen, 2UI fie ifn exblidt - D Simmel, Den fie riebt, Der ift ein Mienich!

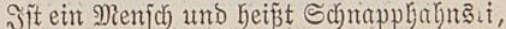
2uf Dex gropien Retirade Sam ex ithr vorbeigelaufen Eintes Miorgens int Siebinge.

Seldemunalück rübrt bie Weiber, Und im 2(ntlib unjres Selden \&ag, wie immer, der Finanznot Blaffe $\Re$ Whmut, Düftre Sorge. 
Seine ganze firiegesfaīe, 3meiumbzwanzig Silbergroicfen, Die er mitgebrad)t nach Spanien, शुard bie Beute Giparteros 1 .

Nicbt eimmal bie ubr gerettet! Błieb zurï̋ In Dem Reihhaus. War ein Erbftür, Softbar und von edjtem Silber.

Unto ex lief mit langen Beinen. 2lber, unbemuist, im saufen Sat er Befferes gemonnen, 2(r bie befte Sd)lad)t - ein 5erz!

Jึ, fie liebt ihn, ihn, Den Erbfeind! $D$, Der unglüićfel'gen $B$ ärin!

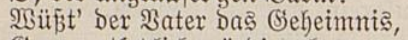
S'anz ent\{ę̧lich) würd' er brummen.

(S) leich Dem alten Dopardo, Der mit Bürgerftolz exDoldyte Die Emilta Salotti, Würbe auch) ber 2Atta Iroll

Seine Iodter lieber töten, Töten mit Den eignen Taben, 2lla exlauben, baỉ fie fänfe In die 2 rme eines \$rinzen!

Doch) in Diejem 2(ugenblicfe Jift er weich geftimmt, hat feine ¿ujt, zu brechen eine Roje, (sh)' Der Sturmmind fie entblättert. ${ }^{2}$

Weich) geftimmt liegt 2 (tta $\mathfrak{I}$ roll In Der Şb̈hle bei Den Seinen.

1 Don Baldomero (5gpartero (1792-1879), beriibmter ipantid)er (Seneral und Mrebrere Jabre Iang Regent von Spanien, nabm in Dem firieg Maria (5)riftinas gegen Don Sarlos eifrig Die \$artei Der eriterent und erfod)t man= d)en Elieg uiber Die fiarlijten.

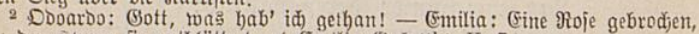
ehe Der Eturm fie entblättert. ("Emilta Ealotti“, V, 7.) 
ฐ̄bn bejchleidyt, wie Tobeşahmuna, Irübe Sehnjucht nach Dem Jenjeits!

"Sinder!" - feufat ex, uno es triefen જ̧(öblich) feine grofien 2(ugen Sinder! meine Erdenmallfahrt "šft vollbrad)t, wix müffen jicheiben.

,̧ente Mittag fam im (d)lafe Mir ein Iraum, ber jefr bedeutjam.

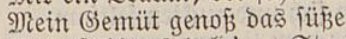
Borgefühl Des bald'gen Sterbens.

,Bin fümmakr niçst abergläubijch, Sin fein fajelbär - Doch gibt es Dinge zmijchen Ero' und Simmel, Die Dem Denfer unerflärlich.

„über Welt und Scjidfal grübelno, 'ָar ich gähnend eingejchlafen, 2Uls mix träumte, dẩ ich) läge Inter einem gropen Baume.

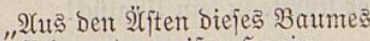
Iroff hemunter meif̈er 5onig, (s)litt mir jujt ins dffne Miaul,

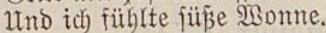

Selig blinzelno in die 5öhe, ๔ah ic) in bes Baumes 2 sipfel (stma fieben fleine Bärchen, Die Dort auf und nieder rutjojten.

3arte, zierliche (seichöpfe, Deren \$elz von rojentoter Farbe war uns an ben Scultern Seibig floctte wie zmei Flïglein.

IĨa, wie jeione Fłlüglein Gatten Dieje rojentoten Bürchen, Into mit überirbijch feinen Jllitenjtinmten jangen fie!

5reine. Stta Troul. 
, 1 ie fie jangen, murbe eisfalt Meine 5aut, Doc) aus ber 5aut fubr Mir die Seel', gleich einer Jllamme; Strablend fiteg fie in ben Simmel."

Urjo iprach mit bebend meichem Sirunzton 2ltta Iroll. Fr fajmieg (5ine Weile, mebmutsvoll Uber jeine Dbren plöbzlich

Spibiten fich und zuditen jeltiant, und empor vom sager fprang er, Freubezitternd, freudebrüllend: "Rinder, hört ifr bieje Raute?

"Ijit das nicht die füpe Stimme (5)rer Mutter? D, ich fenne Das Sebrumme meiner Mumma! Mumma! meine idywarze Mamma!"

Ytta Iroll mit biejen Worten Stürzte mie'n Berrüidter fort

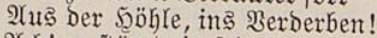
2(d)! ex ftürzte in jein Unglü̈ć!

\section{茎aput XXIV.}

In Dem Thal von Ronceval, YUif bemjelben \$las, mo meilans Des Raroli Magni Reffe Seine Seele auggerödjelt,

Dorten fiel aud) Ytta TroIf, Fiel burd Sinterhalt, wie jenter, Den ber ritterliçe Jubas, S゙anelon von Mainz, vervaten.

U(d)! Das (s) belite im Bären, Das Siefühl Der Śattenliebe, Mুard ein Jallitrid, Den Urafa sijtig ou bemusen wubte. 
Das Siebrumm ber farmarzen Mumma Sat fie nad)geäfft fo täujajeno, Dá̃ Der 2 tta Iroll gelodt maro 2ในล Der fichern Bärentb̈ble -

Wie auf Sehnjuchtafflügeln lief er Durch das Thal, ftand zärtlich fanoperno Manchmal ftill vor einem Feljen, SSlaubt, Die Mumma fei veritedt Dort -

2(c)! veritecft mar bort \&aß̧aro Mit ber Flinte; Diejer f(jofi ifn Mitten Durch bas froke SerzDuoll Gervor ein roter Blutitrom.

Mit Dem Ropfe macfelt' ex Ein'gemal, Doch endich ftüržt' er Stöhnend nieber, zudfte grä̈rich "Miumma!" mar fein leţter Selifzer.

Allo fiel Der edle Seld. Irrjo ftarb er. Dod unjterblid) Nach Dem Tobe auferftehn Wird ex in Dem Ried Des Didjter:

Ưferitehn mirb er im siede,

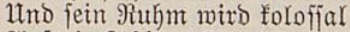

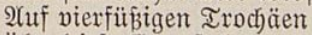
über Dieje Erbe ftelzen.

Der ******* jebt ifm Jn Mুalkalla einjt ein Denfmal, Uno Darauf, im Sapibarfilil, auch bie Jnjurift:

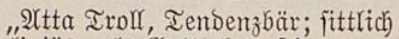
Religiös; ale Ǵatte brünftig; Durch Berführtiein von Dem Beitgeift, Mুaldurjprünglich Sanß̧ülotte;

"Sehr \{chlecht tanzend, Doch Sefinnung Iragend in Dex zott'gen 5odbbruft; Mand)mal aud geftunfen habend; Sein Talent, Doch ein (Eharafter!" 


\section{æaput XXV.}

Dreiundoreibia alte $\mathfrak{B}$ eiber, 2(uf bent 5aupt bie fcharlad)rote 2utbasfefifche Rapuze,

Standen an Des Dorfes Eingang.

Fine butnter, wie Debora, Schlug das Iamburin und tanzte. IIno fie fang Dabei ein \&oblied

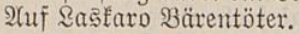

Bier gemalt'ge Piänter trugen İm Iriumph Den toten Bären;

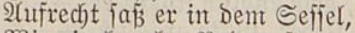
Wie ein franfer Babegajt.

Sinterorein, wie 2 nnerwands Des Berftorbnen, ging \&asfaro Mit Urafa; bieje grïfite Rechts unb linfs, Doch fehr verlegen.

Dex MDjunft bes Maires bielt Eine Rede vor Dem Rathaus, 2tra ber Bug Dorthin gelangte, Und ex iprach von vielen Dingen -

Wie 子. 3. von bem 2(ufichmung Der Marine, von Der \$reffe, \$on ber Runfelrübenjrage,

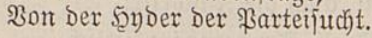

Die Beroienjte IUbwig Philipps Reichlich auseinanderjetseno, J゙ing er über zu Dem Bären Uno ber Sirofithat Des ¿asfaro.

,Du, Sasfaro!" - vief ber Rebner, und ex wij(j) fich Den Schmeip ab Mit Der trifoloren Schärpe „Du, Ząfaro! Du, Ząfaro! 
„Der Du Tranfreid uns .5ijpanien ßon Dem 2Atta Iroll befreit baft, Du bift beiber Ränder Seld, \$nrenäen = 2afayette!"

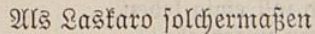
Dffiziell fich) rühmen hörte, Racbte er vergnügt im Barte Und errötete vor Freube,

Und in abgebrochnen sauten, Die fitch jeltjam überfïuzzten, Sat er jeinen Danf geftottert Für bie grofie, grofie ङ5re!

Mit ßermundung blicte jeber

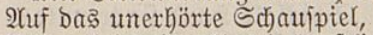
uno geheimmison lf uno ëngitlict) Mumelten Die alten Seiber:

„Der Saڤfaro hat gelacht! Der \&asfaro hat errötet!

Der Iaßfaro hat geiprodien! Er, Der tote Sofn Der Sere!" -

Selb'gen Iags maxd ausgebälgt 2Ytta Iroll und ward veriteigert Seine Saut. Für Gundert Frtanfen

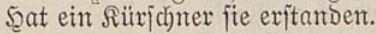

\$ुHundericjön ftaffierte biejer Innd verbrämte fie mit Scharlach, Into verbandelte fie weiter Jür Das Doppelte Des \$reijes.

Erit aus britter Sand befam fie Juliette ${ }^{1}$, und in ihrem ङchlafgemache zut Pari

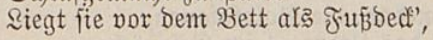

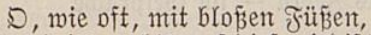
Stand ich nachts auf biejer irdijch

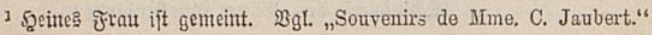


Braunen 5ïlle meines 5elden, 2Uu Der 5̧aut Des 2Ytta Iroll!

Und von \$ebmut tief ergriffer, Dacht' ich Dann an Schillers 2 orte: \$ָa im Rieb foll emig leben, Mū im ฉeben untergehn! 1

\section{Faput XXVI.}

Ind bie Mumma? $2(c 5$, bie Numma J̊ft ein $\mathfrak{W e i b ! ~ ( S e b r e c h l i c h f e i t ~}$

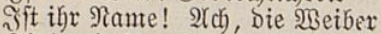
Sind wie \$orzellan gebrechlich.

Arb bes Sdjidfala band fie trennte Bon Dem glorreich edlen Satten, Starb fie nicht des fiummertodes, (sing fie nicht in Trübfinn unter -

Rein, in Ssegenteil, fie jebte Suptig fort ifr Reben, tanzte Rach wie vor, beim \$ublifo Buhlend um den Iagesbeifall.

Fine fefte Stellung, eine ¿ebenslängliçe ßerjorgung, Şat fie endich ơ \$aris כัm ฐarbin Des \$lantes gefunden.

$\mathfrak{A}(\mathrm{S}$ ich) Dorten vor'gen Sonntag Mich erging mit Jultietten,

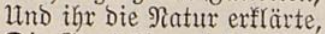
Die Siemächje und bie Beftien,

Die Sirtraffe und bie Beder 3on dem sibanon, das grop̃e Dromedar, die Soldfajanen, 2ud) Das Zebra - im (sejpräche

1 , $\mathfrak{B a s}$ unjterblid) im (Bejang joll Iebent, "PuB́ im Seben untergel)n."

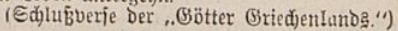


Blieben wir am Ende ftehen 2n ber Brüjtung jener Sinbe, $\mathfrak{B O}_{0}$ die 3 Bären refitoieren Seil'ger Şerr, waอ̉ jafn wir bort!

Ein gemalt'ger 2 iütenbär Ius Sibirien, idnneemeinhaarigt, Spielte Dort ein ïberzartę ¿iebesjpiel mit einer Bärin.

Dieje aber war bie Mumma! War bie Śattin 2Ytta Irolls! Эd) erfannte fite am zärtlich)

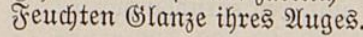

ฐa, fie mar es! Sie, bes Sübent Schmarze Todter! Sie, bie Mumma, Rebt mit einem Ruffen jest, sinem norbijchen Barbaren!

Schmunzelnd iprach zu mix ein Neger, Der zu uns herangetreten: "(Jibt es mohl ein icjönres Sdyaufpiel,

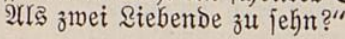

Эch, entgegnete: ,Mit mem Ђab' ich Gier bie E'br' zu 'prechen?" Э̄enter aber rief vermundert: "Rennen Sie mich) gar nidjt mieber?

,Ǐch) bin ja ber Mobrenfürít, Der bei Freiligrath getrommelt. Damal's ging's mix jolecht, in Deutichland Fano ich mich jefr ijoliert.

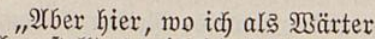
2rngeftellt, wo ich Die \$flanzen Meines Tropenvaterlandes Und auch) হöm' und Iiger finde:

, Sier ijt mir gemütlich mofler, 2uls bei eud auf beutidjen Mefien, Wo icf) tägliç) trommelı muste Und fo folflecht gefüttert wurde! 
,5ab' mich jüngjt vermählt mit einer Blonben Röchin aus Dem Effấ. Sanz und gar in ifren 2 rmen Wiro mix heimatlich) zu Mute!

,Ifre J̈̈̈ве mahnen mid 2ln Die holden Erefanten.

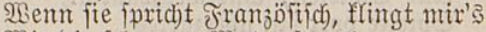
Wie die [chmarze Mitteriprache.

,9) iand)mal feift fie, und idj Denfe 2(n Das Fafieln jener Irommel, Die mit Sajabeln war behangen; Sd)lang' und Seu entflobn Davor. ${ }^{1}$

"Dody im Mondichein jehr empfindjam Sheint tie wie ein Rrofobil, Das สus lauem Strom hernorblidft, Unm bie Sïhle zu genieß̃en. ${ }^{2}$

"ITnd fie gibt mix gute Biffen! Эc) gebeif'! Mit meinem alten, S(frifanijchen 2(pp'tit,

Mie am Niger, freff' ich mieder!

"5ab' mix fchon ein rundes Bäuchlein 2nngemäftet. 2(us Dem 5embe Schaut's hernor, wie'n ichwarzer Mrond, Der aus meipen \$ু心olfen tritt."

\section{ฐaษนt XXVII.}

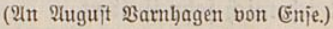

,200 Des 5immels, Meifter subwig, Sabt Jुhx all bas tolle Beug 2lufgegabelt?" Dieje \$isorte Rief Der Rarbinal von Ejte,

1 . (5ig fleudit Der Qeu, es fliebn bie Ed)langen

Bor Dem Rajieln Der Irommel, mit Edjảbeln bebangen."

2 , Yuts Dem laten etrom bliat Dus firotodil,

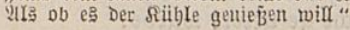


2Ulక ex Das (Sebid)t gelejen ßon bes Rolands Rajereien, DaG 2 rriojto untertbänig Seiner Eminenz gewiomet.

Ja, হarnhagen, alter Freuno, Ia, ich jeh' um beine sippen Jajt biejelben 130 orte ichmeben, g)it bemjelben feinen ä̈cjeln.

Manchmal lacbit bu gar im sejen! Doch) mitunter mag fich ernithaft Deine bohe Stime furchen, Ind (Exinmung überichleid)t bid): -

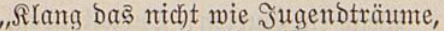
Die $i$ ch träumte mit (Ehamififo Ind Brentano und Fouqué In Den blauen Mondicheinnäch)ten?

,Ij̈t bas̊ nicht baక fromme ¿äuten

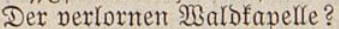
Rlingelt jobalfhaft nicht Dazmij(ben Die befannte Scjellenfappe?

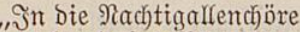
Bricht herein Der Bärenbrummbafi, Dumpf und grollent, Diejer mechjelt SWieder ab mit Seifter!ijpeln!

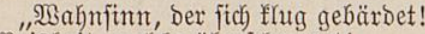
Meisheit, welche überjchnappt! Sterbejeufzer, melche plöblich Sidb) verwandeln in Sselächter!" . . .

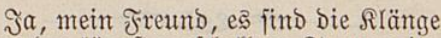
2(us ber längit verichollnen Iraumzeit; গux Daf́ oft moderne Iriller Siaufeln buxch ben alten (sirumbton.

Iroj Des übermutes mirit bu 5ie und bort \$erzagnis jpüren Deiner moblerprobten Milde Sei empfohlen dies Ǵedidyt! 
2(d), es ift vielleidyt bas leste Freie Wals lies ber Romantif! In bes Tages Brand = und Schlachtlärm शुGird es fümmerlich verbalfen.

Indre Beiten, anore ßögel!

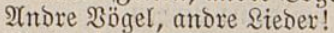
פ̉eld ein Schnattern, wie von (Sä̈njen, Die bą Rapitol gerettet!

W3eld) ein 3witichern! Das fins Spaten, Bfennigglicftchen in Den firallen;

Sie gebärden jich wie Jovis 2loler mit bem Donnerfeil!

Welch ein (Surren! Iurteltauben, Siebejatt, fie mollen baffen, Und binfüro, ftatt ber Benus, গux Belfonas \$̇agen zieben!

Welch ein Sumjen, melterjoütterno!

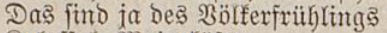
Rolofiale Mraienfäfer, 刃on Berjenfermut ergriffen!

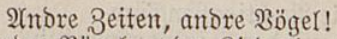
2lnore Bögel, anore sieber! Sie gefielen mir vielfeidgt, פ⿹enn ich andre Dhren hätte!

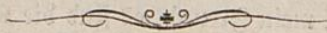

Druderei bes Bibliographifđen Inftituts, 厄arl Deyer. - Leipzig. 


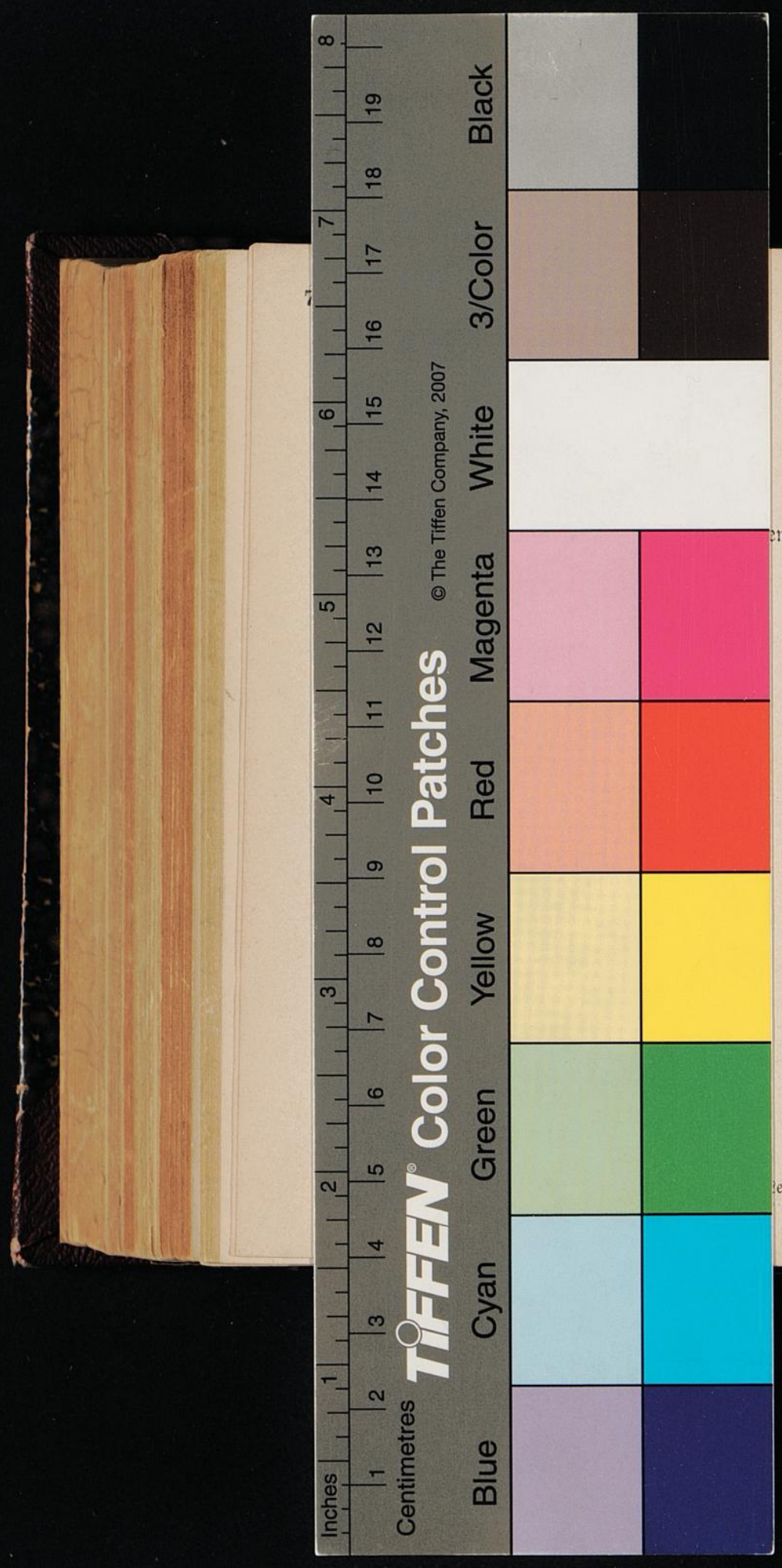

